\title{
Diffusion Data for Silicate Minerals, Glasses, and Liquids
}

\author{
John B. Brady
}

\section{INTRODUCTION}

Diffusion is an integral part of many geologic processes and an increasing portion of the geologic literature is devoted to the measurement, estimation, and application of diffusion data. This compilation is intended to be a guide to recent experimentallydetermined diffusion coefficients and, through the papers cited, to important older literature. To provide a context for the tables, a brief sumniary of the equations required for a phenomenological (macroscopic) description of diffusion follows. Although the equa tions for well-constrained experiments are relatively straightforward, the application of the resulting diffusion coefficients to complex geologic problems may not be straightforward. The reader is urged to read one or more diffusion texts [e.g., 99, 121, 32, 147, 135] before attempting to use the data presented here.

\section{FORCES AND FLUXES}

Diffusion is the thermally-activated, relative movement (flux) of atoms or molecules that occurs in response to forces such as gradients in chemical potential or temperature. Diffusion is spontaneous and, therefore, must lead to a net decrease in free

J. B. Brady, Department of Geology, Smith College, Northampton, MA 01063

Mineral Physics and Crystallography

A Handbook of Physical Constants

AGU Reference Shelf 2

Copyright 1995 by the American Geophysical Union. energy. For example, the movement of $\Delta n_{i}$ moles of chemical component $\mathrm{i}$ from a region (II) of high chemical potential $\left(\mu_{\mathrm{i}}{ }^{\mathrm{II}}\right)$ to a region (I) of lower chemical potential $\left(\mu_{\mathrm{i}}^{\mathrm{I}}\right)$ will cause the system Gibbs energy $(G)$ to fall since

$$
\Delta G^{I}+\Delta G^{I I}=\Delta n_{i}\left(\frac{\partial G^{l}}{\partial n_{i}}\right)_{P, T, n_{j}}-\Delta n_{i}\left(\frac{\partial G^{I I}}{\partial n_{i}}\right)_{P, T, n_{j}}
$$

and using the definition of $\mu_{\mathrm{i}}[139, \mathrm{p} .128]$,

$$
\Delta \mathrm{G}^{\text {Total }}=\Delta \mathrm{n}_{\mathrm{i}}\left(\mu_{\mathrm{i}}^{\mathrm{I}}-\mu_{\mathrm{i}}^{\text {II }}\right)<0 .
$$

Thus, a chemical potential gradient provides a therm odynamic force for atom movement.

On a macroscopic scale, linear equations appear to be adequate for relating each diffusive flux to the set of operative forces [137, p.45]. The instantaneous, one-dimensional, isothermal diffusive flux $\mathrm{J}_{i}^{\mathrm{R}}$ (moles of $i / m^{2} s$ ) of component $i$ in a single-phase, $n$ component system with respect to reference frame $R$ may be described by

$$
\mathbf{J}_{i}^{R}=\sum_{j=1}^{n}-L_{i j}^{R}\left(\frac{\partial \mu_{j}}{\partial x}\right),
$$

where $x(m)$ is distance and the $n^{2}$ terms $L_{i j}^{R}$ (moles of $\mathrm{i} / \mathrm{m} \cdot \mathrm{J} \cdot \mathrm{s})$ are "phenomenological diffusion coefficients" [36]. Because each component of the system may move in response to a gradient in the chemical potential of any other component, the complexity of describing diffusion in multicomponent systems rises 
rapidly with the number of components. Two impor tant results help limit this complexity. First, the isothermal, isoharic Gibhs-Duhem equation $[139, \mathrm{p}$. 134]

$$
\sum_{i=1}^{n} n_{i} d \mu_{i}=0
$$

for the single phase in which the diffusion occurs reduces the number of independent gradients to $(n-1)$ and the number of diffusion coefficients to $(n-1)^{2}$. Second, Onsager [132, 133] showed that if the forces, fluxes, components, and reference frame are properly chosen, the matrix of coefficients relating the torces and fluxes is symmetrical

$\mathrm{L}_{\mathrm{ij}}^{\mathrm{R}}=\mathrm{L}_{\mathrm{ji}}^{\mathrm{R}}$,

reducing the number of independent diffusion coefficients to $(2 n-1)$.

Although equations (3)-(5) are theoretically satisfying, they are not generally used to describe diffusion experiments, in part because chemical potential gradients are not directly measurable in most cases. Fisher [51], Joesten [94, 96, 97], and others [e.g., $55,98,16,1]$ have applied these equations successfully in modeling the diffusion evolution of coronas and other textures in some rocks. However, most workers use empirical equations related to (3) that involve measurable compositions $C_{j}$ (moles of $\mathrm{j} / \mathrm{m}^{3}$ )

$$
\mathrm{J}_{\mathrm{i}}^{\mathrm{R}}=\sum_{\mathrm{j}=\mathrm{l}}^{\mathrm{n}-1}-\mathrm{D}_{\mathrm{ij}}^{\mathrm{R}}\left(\frac{\partial \mathrm{C}_{\mathrm{j}}}{\partial \mathrm{x}}\right)
$$

[134]. Only (n-1) compositions are independent, but unfortunately $D_{i j}^{R} \neq D_{j i}^{R}$ so that $(n-1)^{2}$ diffusion coefficients $D_{i j}^{R}\left(\mathrm{~m}^{2} / \mathrm{s}\right)$ are needed for $(6)$.

The most obvious simplification of (6) is to limit the number of components to 2 and, therefore, the number of required diffusion coefficients to 1 . Most experimentalists achieve this by their experimental design. The next two sections present definitions and equations used to describe these binary (2-component) experiments. Additional ways to simplify the treatment of multicomponent systems are addressed in Section 5.

\section{FICK'S LAWS}

Adolf Fick's [50] empirical equations were used to describe binary diffusion experiments long before the more general equations (3) and (6) were developed. Fick's First Law

$$
\mathrm{J}_{\mathrm{i}}=-\mathrm{D}_{\mathrm{i}}\left(\frac{\mathrm{dC_{ \textrm {i } }}}{\mathrm{dx}}\right)
$$

relates the instantaneous flux $\mathrm{J}_{\mathrm{i}}$ (moles of $\mathrm{i} / \mathrm{m}^{2} \mathrm{~s}$ ) of component $i$ to the one-dimensional gradient of the concentration of $\mathrm{i}, \mathrm{dC}_{\mathrm{i}} / \mathrm{dx}$ (moles of $\left.\mathrm{i} / \mathrm{m}^{4}\right)$, and defines the diffusion coefficient $D_{i}\left(\mathrm{~m}^{2} / \mathrm{s}\right)$. However, unless the experiment attains a stcady statc, time $(\mathrm{t})$ is also a variable and a continuity equation (Fick's Second Law)

$\left(\frac{\partial \mathrm{C}_{\mathrm{i}}}{\partial \mathrm{t}}\right)_{\mathrm{x}}=\left(\frac{\partial}{\partial \mathrm{x}}\left[\mathrm{D}_{\mathrm{i}}\left(\frac{\partial \mathrm{C}_{\mathrm{i}}}{\partial \mathrm{x}}\right)_{\mathrm{t}}\right]\right)_{\mathrm{t}}$

must be solved. If $D_{i}$ is not a function of composition $\left(C_{i}\right)$ and, therefore, not a function of position ( $x$ ), then equation (4) may be simplified to

$$
\left(\frac{\partial C_{i}}{\partial t}\right)_{x}=D_{i}\left(\frac{\partial^{2} C_{i}}{\partial x^{2}}\right)_{t},
$$

which has many analytical solutions $[6,99,17,32]$.

Some commonly used solutions to (5) for planar geometries are given in Table 1 and two of these are shown graphically in Figure 1. All of the equations (7)-(13) implicitly assume constancy of volume, for which the fixed laboratory reference frame is a mean volume reference frame, and must be modified if the sample volume does change [10]. Similar analytical solutions exist for related boundary conditions and/or other geometries, notably spherical and cylindrical cases. More complicated boundary conditions and geometries may require numerical approximation $[32$, Chap. 8].

It is clear from Table 1 and Figure 1 that the parameter $\sqrt{D_{i} t}$ may be used to characterize the extent of diffusion. In semi-infinite cases such as (10) and (11), the distance $x$ that has attained a particular value of $C_{i}$ after time $t$ is proportional to $\sqrt{D_{i} t}$. In finite 
Table 1. Commonly-used solutions to equation (9)

\begin{tabular}{|c|c|c|}
\hline Boundary Conditions & Solution & \\
\hline $\begin{array}{l}\text { Thin-film Solution } \\
\left\{\begin{array}{l}C_{i} \rightarrow C 0 \text { for }|x|>0 \text { as } t \rightarrow 0 \\
c_{i} \rightarrow \infty \text { for } x=0 \text { as } t \rightarrow 0\end{array}\right\}\end{array}$ & $\left(\frac{C_{i}-C 0}{\alpha}\right)=\frac{1}{2 \sqrt{\pi D_{i} t}} \exp \left(\frac{-x^{2}}{4 D_{i} t}\right)$ where $\alpha \equiv \int_{-\infty}^{+\infty}\left(C_{i}-C 0\right) d x$ & (10) \\
\hline $\begin{array}{l}\text { Semi-infinite Pair Solution } \\
\left\{\begin{array}{l}\mathrm{C}_{\mathrm{i}}=\mathrm{C} 0 \text { for } \mathrm{x}>0 \text { at } \mathrm{t}=0 \\
\mathrm{C}_{\mathrm{i}}=\mathrm{Cl} \text { for } \mathrm{x}<0 \text { at } \mathrm{t}=0\end{array}\right\}\end{array}$ & $\left(\frac{C_{i}-C 0}{C l-C 0}\right)=\frac{1}{2} \operatorname{erfc}\left(\frac{x}{2 \sqrt{D_{i} t}}\right)$ & (11) \\
\hline $\begin{array}{l}\text { Finite Pair Solution } \\
\left\{\begin{array}{l}\mathrm{C}_{\mathrm{i}}=\mathrm{C} 1 \text { for } 0<\mathrm{x}<\mathrm{h} \text { at } \mathrm{t}=0 \\
\mathrm{C}_{\mathrm{i}}=\mathrm{C} 0 \text { for } \mathrm{h}<\mathrm{x}<\mathrm{L} \text { at } \mathrm{t}=0 \\
\left(\partial \mathrm{C}_{\mathrm{i}} / \partial \mathrm{x}\right)_{\mathrm{t}}=0 \text { for } \mathrm{x}=0 \& \mathrm{~L}\end{array}\right\}\end{array}$ & $\left(\frac{C_{i}-C 0}{C l-C 0}\right)=\frac{h}{L}+\frac{2}{\pi} \sum_{n=1}^{\infty} \frac{1}{n} \sin \left(\frac{n \pi h}{L}\right) \exp \left(\frac{-D_{i} n^{2} \pi^{2} t}{L^{2}}\right) \cos \left(\frac{n \pi x}{L}\right)$ & (12) \\
\hline $\begin{array}{l}\text { Finite Sheet - Fixed Surface Composition } \\
\left\{\begin{array}{l}\mathrm{C}_{1}=\mathrm{C} 0 \text { for }-\mathrm{L}<\mathrm{x}<\mathrm{L} \text { at } \mathrm{t}=0 \\
\mathrm{C}_{\mathrm{i}}=\mathrm{Cl} \text { for } \mathrm{x}=0 \& \mathrm{~L} \text { at } \mathrm{t}>0\end{array}\right\}\end{array}$ & $\left(\frac{C_{i}-C 0}{C l-C 0}\right)=1-\frac{4}{\pi} \sum_{n=0}^{\infty} \frac{-1^{n}}{2 n+1} \exp \left(\frac{-D_{i}(2 n+1)^{2} \pi^{2} t}{4 L^{2}}\right) \cos \left(\frac{(2 n+1) \pi x}{2 L}\right)$ & (13) \\
\hline
\end{tabular}

cases such as (12) and (13), the fractional extent of completion of homogenization by diffusion is proportional to $\sqrt{D_{i} t}$. These $\sqrt{D_{i} t}$ relations provide important tests that experimental data must pass if diffusion is asserted as the rate-controlling process. They also provide simple approximations to the limits of diffusion when applying measured diffusion coefficients to specific problems [147].

\section{DIFFUSION COEFFICIENTS}

In general, one must assume that $\mathrm{D}_{\mathrm{i}}$ is a function of $C_{i}$. Therefore, equations (9)-(13) may be used with confidence only if $C_{i}$ does not change appreciably during the experiment. This is accomplished either (a) by using a measurement technique (typically involving radioactive tracers) that can detect very small changes in $C_{i}$, or (b) by using diffusional exchange of stable isotopes of the same element that leave the element concentration unchanged. Approach (a) yields a "tracer diffusion coefficient" for the element that is specific to the bulk composition studied. Approach (b) yields a "self-diffusion coeffi cient" for the isotopically doped element that is also specific to the bulk chemical composition. Both approaches generally ignore the opposite or exchange flux that must occur in dominantly ionic phases such as silicate minerals, glasses, and liquids.

If $\mathrm{C}_{\mathrm{i}}$ does change significantly in the experiment and $\mathrm{D}_{\mathrm{i}}$ is a function of $\mathrm{C}_{\mathrm{i}}$, observed compositional profiles might not match the shape of those predicted by (9)-(13). In such cases the $D_{i}$ calculated with these equations will be at best a compositional "average" and equation (8) should be considered. Experiments in which composition does change significantly are often termed "interdiffusion" or "chemical diffusion" experiments. A commonly-used analytical solution to (8), for the same boundary conditions as for equation (11), was obtained by Matano [122] using the Boltzmann [8] substitution $(\chi \equiv \mathrm{x} / \sqrt{\mathrm{t}})$ :

$$
D_{i}(C 2)=\frac{-1}{2 t}\left(\frac{d x}{d C_{i}}\right)_{C_{i}=C 2} \int_{C_{i}=C 0}^{C_{i}=C 2} x d C_{i}
$$

Equation (14) can be evaluated numerically or graphically from a plot of $\left(\mathrm{C}_{\mathrm{i}}-\mathrm{C} 0\right) /(\mathrm{C} 1-\mathrm{C} 0)$ versus $\mathrm{x}$, where the point $x=0$ (the Matano interface) is selected such that

$$
\int_{C_{i}=C 0}^{C_{i}=C l} x d C_{i}=0
$$



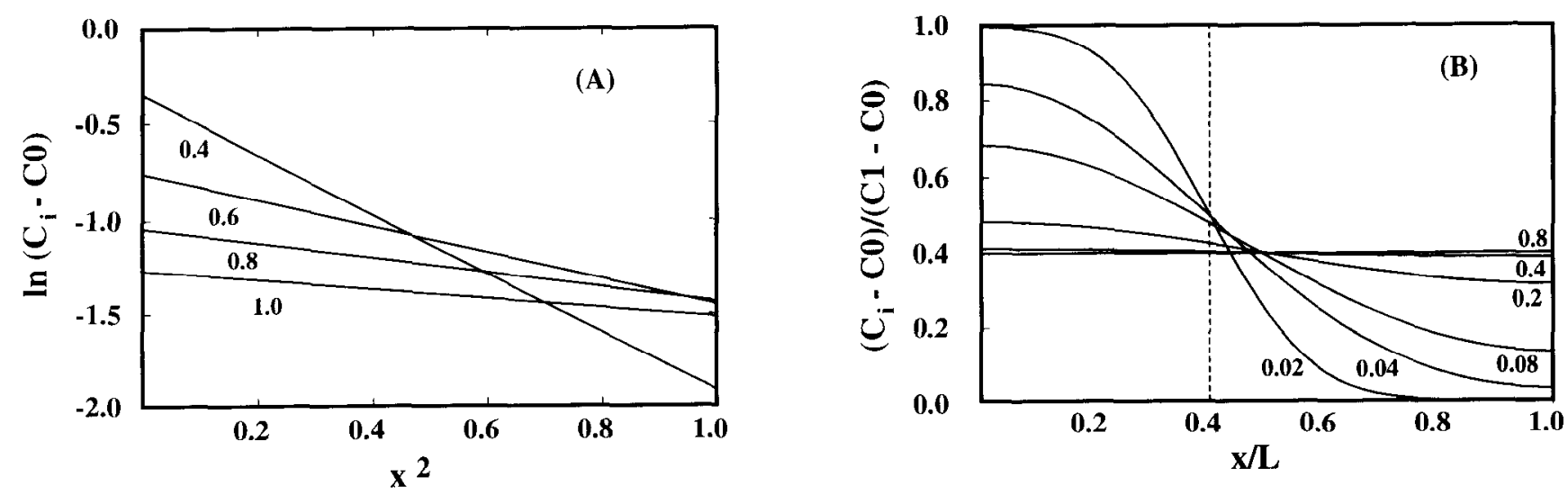

Figure 1. Graphical solutions to equation (9). (A) The "thin film" solution of equation (10) is shown with $\alpha=1$ for various values of $\sqrt{D_{i} t}$ (labels on lines). Plotting $\ln \left(C_{i}-C 0\right)$ as a function of $x^{2}$ at any time yields a straight line of slope $-1 /\left(4 D_{i} t\right)$. (B) The "finite pair" solution of equation (12) is shown for $h / L=0.4$ and various values of $D t / L^{2}$ (labels on curves). Plotting $\left(C_{i}-C 0\right) /(C 1$ $\mathrm{C} 0$ ) as a function of $\mathrm{x} / \mathrm{L}$ normalizes all cases to a single dimensionless graph. The initial boundary between the two phases is marked by a dashed vertical line.

[32, p.230-234]. For binary, cation exchange between ionic crystals, the Matano interface is the original boundary between the two crystals. Diffusion experiments that follow the Boltzmann-Matano approach have the advantage of determining $D_{i}$ as a function of $\mathrm{C}_{\mathrm{i}}$ and the disadvantage of risking the complications of multicomponent diffusion, for which neither (8) nor (14) is correct.

Darken [35] and Hartley and Crank [83] showed that for electrically neutral species, the binary $(A \leftrightarrow B)$ interdiffusion coefficients $\left(\mathrm{D}_{\mathrm{AB}_{-1}}\right)$ obtained in a Boltzmann-Matano type experiment and the tracer diffusion coefficients $\left(D_{A}^{*}\right.$ and $D_{B}^{*}$ ) for the interdiffusing species are related by

$$
\mathrm{D}_{\mathrm{AB}_{-1}}=\left(\mathrm{N}_{\mathrm{B}} \mathrm{D}_{\mathrm{A}}^{*}+\mathrm{N}_{\mathrm{A}} \mathrm{D}_{\mathrm{B}}^{*}\right)\left[1+\left(\frac{\partial \ln \gamma_{\mathrm{A}}}{\partial \ln \mathrm{N}_{\mathrm{A}}}\right)_{\mathrm{P}, \mathrm{T}}\right] \text {, }
$$

where $\mathrm{N}_{\mathrm{A}}$ is the mole fraction and $\gamma_{\mathrm{A}}$ the molar activity coefficient of component $\mathrm{A}$. Darken developed his analysis in response to the experiments of Smigelskas and Kirkendall [148], who studied the interdiffusion of $\mathrm{Cu}$ and $\mathrm{Zn}$ between $\mathrm{Cu}$ metal and $\mathrm{Cu}_{70} \mathrm{Zn}_{30}$ brass. Smigelskas and Kirkendall observed that Mo wires (inert markers) placed at the boundary between the $\mathrm{Cu}$ and brass moved in the direction of the brass during their experiments, indicating that more $\mathrm{Zn}$ atoms than $\mathrm{Cu}$ atoms crossed the boundary. Darken's analysis showed that in the presence of inert markers the independent fluxes and, therefore, the "tracer" diffusion coefficients of $\mathrm{Cu}$ and $\mathrm{Zn}$ can be determined in addition to the interdiffusion coefficient $\mathrm{D}_{\mathrm{CuZn}_{-1}}$.

A similar analysis for binary cation interdiffusion $\left(\mathrm{AZ} \leftrightarrow \mathrm{BZ}\right.$ where $\mathrm{AZ}$ represents $\mathrm{A}_{\mathrm{z}}^{+\mathrm{a}} \mathrm{Z}_{\mathrm{a}}^{-\mathrm{z}}$ and $\mathrm{BZ}$ represents $B_{z}^{+b} Z_{b}^{-z}$ ) in appreciably ionic materials such as silicate minerals yields $[5,112,113,10]$ :

$$
\begin{aligned}
& \mathrm{D}_{\mathrm{A}_{\mathrm{b}} \mathrm{B}_{-\mathrm{a}}}=\left[\frac{\left(\mathrm{D}_{\mathrm{AZ}}^{*}\right)\left(\mathrm{D}_{\mathrm{BZ}}^{*}\right)\left(\mathrm{aN}_{\mathrm{AZ}}+\mathrm{bN}_{\mathrm{BZ}}\right)^{2}}{\left(\mathrm{a}^{2} \mathrm{~N}_{\mathrm{AZ}} \mathrm{D}_{\mathrm{AZ}}^{*}+\mathrm{b}^{2} \mathrm{~N}_{\mathrm{BZ}} \mathrm{D}_{\mathrm{BZ}}^{*}\right)}\right][1+ \\
& \left.\left(\frac{\partial \ln \gamma_{\mathrm{AZ}}}{\partial \ln \mathrm{N}_{\mathrm{AZ}}}\right)_{\mathrm{P}, \mathrm{T}}\right],
\end{aligned}
$$

which requires vacancy diffusion if $a \neq b$. If $a=b$, then (17) simplifies to

$$
\begin{aligned}
& \mathrm{D}_{\mathrm{AB}_{-1}}=\left[\frac{\left(\mathrm{D}_{\mathrm{AZ}}^{*}\right)\left(\mathrm{D}_{\mathrm{BZ}}^{*}\right)}{\left(\mathrm{N}_{\mathrm{AZ}} \mathrm{D}_{\mathrm{AZ}}^{*}+\mathrm{N}_{\mathrm{BZ}} \mathrm{D}_{\mathrm{BZ}}^{*}\right)}\right][1+ \\
& \left.\left(\frac{\partial \ln \gamma_{\mathrm{AZ}}}{\partial \ln \mathrm{N}_{\mathrm{AZ}}}\right)_{\mathrm{P}, \mathrm{T}}\right]
\end{aligned}
$$

[121]. This expression permits interdiffusion coefficients to be calculated from more-easily-measured tracer diffusion coefficients. For minerals that are not 
ideal solutions, the "thermodynamic factor" in brackets on the right side of (16)-(18) can significantly change the magnitude (and even the sign!) of interdiffusion coefficients from those expected for an ideal solution $[12,25]$.

\section{MULTICOMPONENT DIFFUSION}

Rarely is diffusion in geologic materials binary. Multicomponent diffusion presents the possibility that diffusive fluxes of one component may occur in response to factors not included in (7)-(18), such as gradients in the chemical potentials of other components, coupling of diffusing species, etc. In these cases, equation (6) or (3) must be used. The offdiagonal ( $i \neq j$ ) diffusion coefficients, $D_{i j}^{R}$ or $L_{i j}^{R}$, are unknown for most materials and most workers are forced to assume (at significant peril!) that they are zero. Garnet is the one mineral for which offdiagonal diffusivities are available $[19,20]$ and they were found to be relatively small.

One approach used by many is to treat diffusion that is one-dimensional in real space as if it were onedimensional in composition space. This approach was formalized by Cooper and Varshneya [28, 30] who discuss diffusion in ternary glasses and present criteria to be satisfied to obtain "effective binary diffusion coefficients" from multicomponent diffusion experiments. In general, diffusion coefficients obtained with this procedure are functions of both composition and direction in composition space. Another approach, developed by Cullinan [33] and Gupta and Cooper [75, 29], is to diagonalize the diffusion coefficient matrix for (6) through an eigenvector analysis. Although some simplification in data presentation is achieved in this way, a matrix of diffusion coefficients must be determined before the analysis can proceed.

Lasaga [105, see also 161] has generalized the relationship (17) to multicomponent minerals. The full expression is quite long, but simplifies to

$$
D_{i j}=D_{i}^{*} \delta_{i j}-\left(\frac{D_{i}^{*} C_{i} z_{i} z_{j}}{\sum_{k=1}^{n} D_{k}^{*} C_{k} z_{k}^{2}}\right)\left(D_{j}^{*}-D_{n}^{*}\right)
$$

if the solid solution is thermodynamically ideal. In (19) $z_{i}$ is the charge on cation $i, \delta_{i j}=1$ if $i=j$, and $\delta_{i j}$ $=0$ if $i \neq j$. This approach, using tracer diffusion coefficients $D_{j}^{*}$ to calculate multicomponent interdiffusion coefficient matrices, and its inverse offer the most hope for diffusion analysis of multicomponent problems $[115,19,20]$. It should be noted in using equations (16)-(19) that the tracer diffusivities themselves may be functions of bulk composition.

\section{EXPERIMENTAL DESIGN}

A few additional considerations should be mentioned regarding the collection and application of diffusion data.

- In anisotropic crystals, diffusion may be a function of crystallographic orientation and the diffusion coefficient becomes a second rank tensor. The form of this tensor is constrained by point group symmetry and the tensor can be diagonalized by a proper choice of coordinate system [131].

- Vacancies, dislocations, and other crystal defects may have profound effects on diffusion rates [e.g., $121,164]$. Therefore, it is essential that the mineral being studied is well-characterized. If the mineral, liquid, or glass contains a multivalent element like $\mathrm{Fe}$, then an equilibrium oxygen fugacity should be controlled or measured because of its effect on the vacancy concentration.

- Diffusion in rocks or other polycrystalline materials may occur rapidly along grain boundaries, crystal interfaces, or surfaces and will not necessarily record intracrystalline diffusivities. Experiments involving grain-boundary diffusion [e.g., 102, 103, $11,47,49,95,154,13,158]$ are beyond the scope of this summary. If polycrystalline materials are used in experiments to measure "intrinsic" or "volume" diffusion coefficients, then the contributions of "extrinsic" grain-boundary diffusion must be shown to be negligible.

- Water can have a major effect on diffusion in many geologic materials, even in small quantities [e.g., 73, 150, 46, 167, 71]. If water is present, water fugacities are an essential part of the experimental data set.

- Because many kinetic experiments cannol be "reversed," every effort should be made to demonstrate that diffusion is the rate-controlling process. Important tests include the $\sqrt{D_{\mathrm{i}} \mathrm{t}}$ relations noted in equations (10)-(13) and the "zero time experiment." The $\sqrt{D_{i} t}$ test can be used if data are gathered at the same physical conditions for at least two times, preferably differing by a factor of four or more. The often-overlooked "zero time experiment," 
which duplicates the sample preparation, heating to the temperature of the experiment, quenching (after "zero time" at temperature), and data analysis of the other diffusion experiments, commonly reveals sources of systematic errors.

These and other important features of diffusion experiments are described in Ryerson [141].

\section{DATA TABLE}

Due to space limitations, the data included in this compilation have been restricted to comparatively recent experimental mcasurements of diffusion cocfficients for silicate minerals, glasses, and liquids. Many good, older data have been left out, but can bc found by following the trail of references in the recent papers that are included. Older data may also be found by consulting the compilations of Freer [56, 57], Hofmann [86], Askill [2], and Harrop [81]. Some good data also exist for important non-silicate minerals such as apatite $[157,23,45]$ and magnetite titanomagnetite $[65,136,59]$, but the silicate minerals offered the clearest boundary for this paper.

Almost all of the data listed are for tracer or selfdiffusion. Interdiffusion (chemical diffusion) experiments involving minerals are not numerous and interdiffusion data sets do not lend themselves to compact presentation. Interdiffusion data for silicate liquids and glasses are more abundant, but are not included [see 156, 3, 4]. Neither are non-isothermal (Soret) diffusion data [see 108]. Diffusion data listed for silicate glasses and liquids have been further restricted to bulk compositions that may be classified as either basalt or rhyolite.

Because diffusion is thermally activated, coefficients for diffusion by a single mechanism at different temperatures may be described by an Arrhenius equation

$$
\mathrm{D}=\mathrm{D}_{\mathrm{o}} \exp \left(\frac{-\Delta \mathrm{H}}{\mathrm{RT}}\right)
$$

and fit by a straight line on a graph of $\log \mathrm{D}\left(\mathrm{m}^{2} / \mathrm{s}\right)$ as a function of $1 / \mathrm{T}\left(\mathrm{K}^{-1}\right)$ [147, Chap. 2]. $\log \mathrm{D}_{\mathrm{o}}$ $\left(\mathrm{m}^{2} / \mathrm{s}\right)$ is the intercept of the line on the $\log \mathrm{D}$ axis $(1 / T=0) . \Delta H /(2.303 \cdot R)$ is the slope of the line where $\mathrm{R}$ is the gas constant $(8.3143 \mathrm{~J} / \mathrm{mole} \cdot \mathrm{K})$ and
$\Delta H(\mathrm{~J} /$ mole $)$ is the "activation energy." $\mathrm{D}_{\mathrm{o}}$ and $\Delta \mathrm{H}$ have significance in the atomic theory of diffusion [see 32,121 ] and may be related for groups of similar materials $[162,82,106]$.

Diffusion data are listed in terms of $\Delta H$ and $\log D_{0}$ and their uncertainties. In many cases data were converted to the units $\left(\mathrm{kJ}, \mathrm{m}^{2} / \mathrm{s}\right)$ and form $\left(\log \mathrm{D}_{\mathrm{o}}\right)$ of this compilation. Logarithms are listed to 3 decimal places for accurate conversion, even though the original data may not warrant such precision. No attempt was made to reevaluatc the data, the fit, or the uncertainties given (or omitted) in the original papers. Also listed arc the conditions of the experiment as appropriate including the temperature range, pressure range, oxygen fugacity, and sample geometry. Extrapolation of the data using (20) to conditions outside of the experimental range is not advisable. However, for ease of comparison $\log \mathrm{D}$ is listed for a uniform temperature of $800^{\circ} \mathrm{C}\left(1200^{\circ} \mathrm{C}\right.$ for the glasses and liquids), even though this temperature may be outside of the experimental range. Use these tabulated $\log \mathrm{D}$ numbers with caution.

Finally, a sample "closure temperature" $\left(T_{c}\right)$ has been calculated for the silicate mineral diffusion data. The closure temperature given is a solution of the Dodson [38] equation

$$
\left(\frac{\Delta \mathrm{H}}{\mathrm{RT}_{\mathrm{c}}}\right)=\ln \left(\frac{-55 \mathrm{RT}_{\mathrm{c}}^{2} \mathrm{D}_{\mathrm{o}}}{\mathrm{a}^{2} \Delta \mathrm{H}(\mathrm{dT} / \mathrm{dt})}\right)
$$

for a sphere of radius $\mathrm{a}=0.1 \mathrm{~mm}$ and a cooling rate $(\mathrm{dT} / \mathrm{dt})$ of $5 \mathrm{~K} / \mathrm{Ma}$. The example closure temperatures were included because of the importance of closure temperatures in the application of diffusion data to petrologic [107] and geochronologic [123, Chap. 5] problems. Note that other closure temperatures would be calculated for different crystal sizes, cooling rates, and boundary conditions.

Acknowledgments. This paper was improved by helpful comments on earlier versions of the manuscript by D. J. Cherniak, R. A. Cooper, R. Freer, J. Ganguly, B. J. Giletti, S. J. Kozak, E. B. Watson, and an anonymous reviewer. N. Vondell provided considerable help with the library work. 


\begin{tabular}{|c|c|c|c|c|c|c|c|c|c|c|c|}
\hline $\begin{array}{l}\text { Mineral, Glass, } \\
\text { or Liquid }\end{array}$ & $\begin{array}{c}\text { Orienta- } \\
\text { tion }\end{array}$ & $\begin{array}{l}\text { Diffusing } \\
\text { Component } \\
\end{array}$ & $\begin{array}{r}\text { Temperature } \\
\text { Range }\left({ }^{\circ} \mathrm{C}\right)\end{array}$ & $\begin{array}{c}\mathrm{P} \\
(\mathrm{MPa}) \\
\end{array}$ & $\begin{array}{c}\mathrm{O}_{2} \\
(\mathrm{MPa}) \\
\end{array}$ & $\begin{array}{c}\Delta \mathrm{E}_{\mathrm{a}} \\
(\mathrm{kJ} / \mathrm{mole})\end{array}$ & $\begin{array}{c}\log \mathrm{D}_{\mathrm{o}}(\text { or } \mathrm{D}) \\
\left(\mathrm{m}^{2} / \mathrm{s}\right) \\
\end{array}$ & $\begin{array}{l}\log \mathrm{D} \\
800^{\circ} \mathrm{C} \\
\end{array}$ & $\begin{array}{l}" \mathrm{~T}_{c} " \\
\left({ }^{\circ} \mathrm{C}\right)\end{array}$ & Experiment/Comments & Ref. \\
\hline $\begin{array}{l}\text { B-quartz } \\
\left(\mathrm{SiO}_{2}\right)\end{array}$ & $\mathscr{H} \mathrm{c}$-axis & $\mathrm{H}$ & $700-900$ & $\begin{array}{l}890- \\
1550\end{array}$ & UB & $\begin{array}{l}200 \\
( \pm 20)\end{array}$ & -0.854 & -10.6 & 188 & $\begin{array}{l}\text { Exchange with water-bearing } \\
\text { fluid, "bulk" IR spectra }\end{array}$ & [104] \\
\hline $\begin{array}{l}\beta \text {-quartz } \\
\left(\mathrm{SiO}_{2}\right)\end{array}$ & // c-axis & ${ }^{18} \mathrm{O}$ & $600-800$ & 100 & UB & $142( \pm 4)$ & $-10.398( \pm 0.272)$ & -17.3 & 282 & $\begin{array}{l}\text { Exchange with }{ }^{18} \mathrm{O} \text {-enriched } \\
\text { water, ion probe profiles }\end{array}$ & {$[68]$} \\
\hline $\begin{array}{l}\beta \text {-quartz } \\
\left(\mathrm{SiO}_{2}\right) \\
\end{array}$ & $\perp$ c-axis & ${ }^{18} \mathrm{O}$ & $600-800$ & 100 & UB & $234( \pm 8)$ & $-8.000( \pm 2.239)$ & -19.4 & 498 & $\begin{array}{l}\text { Exchange with }{ }^{18} \mathrm{O} \text {-enriched } \\
\text { water, ion probe profiles }\end{array}$ & [68] \\
\hline $\begin{array}{l}\beta \text {-quartz } \\
\left(\mathrm{SiO}_{2}\right)\end{array}$ & $/ / c$-axis & ${ }^{18} \mathrm{O}$ & $700-850$ & 100 & $\begin{array}{l}\text { UB/ } \\
\text { NO }\end{array}$ & $138.5( \pm 19.1)$ & $-10.680( \pm 0.955)$ & -17.4 & 278 & $\begin{array}{l}\text { Exchange with }{ }^{18} \mathrm{O} \text {-enriched } \\
\text { water, ion probe profiles }\end{array}$ & [37] \\
\hline $\begin{array}{l}\beta \text {-quartz } \\
\left(\mathrm{SiO}_{2}\right)\end{array}$ & $\perp$ c-axis & ${ }^{18} \mathrm{O}$ & $700-850$ & 100 & $\begin{array}{l}\text { UB/ } \\
\text { NO }\end{array}$ & $203.7(+2.3)$ & $-9.413( \pm 0.151)$ & -19.3 & 462 & $\begin{array}{l}\text { Exchange with }{ }^{18} \mathrm{O} \text {-enriched } \\
\text { water, ion probe profiles }\end{array}$ & [37] \\
\hline $\begin{array}{l}\beta \text {-quartz } \\
\left(\mathrm{SiO}_{2}\right) \\
\end{array}$ & $/ / c$-axis & ${ }^{18} \mathrm{O}$ & $745-900$ & 10 & $\mathrm{CO}_{2}$ & $159( \pm 13)$ & $-3.678( \pm 0.132)$ & -11.4 & 146 & $\begin{array}{l}\text { Exchange with } \mathrm{C}^{18} \mathrm{O}_{2} \text { gas, ion } \\
\text { probe profiles }\end{array}$ & [146] \\
\hline $\begin{array}{l}\alpha \text {-quartz } \\
\left(\mathrm{SiO}_{2}\right)\end{array}$ & $/ / \mathrm{c}$-axis & ${ }^{18} \mathrm{O}$ & $450-590$ & 100 & UB & $243( \pm 17)$ & -4.538 & -16.4 & 389 & $\begin{array}{l}\text { Exchange with }{ }^{18} \mathrm{O} \text {-enriched } \\
\text { water, ion probe profiles }\end{array}$ & [48] \\
\hline $\begin{array}{l}\beta \text {-quartz } \\
\left(\mathrm{SiO}_{2}\right)\end{array}$ & $\perp(101)$ & ${ }^{30} \mathrm{Si}$ & $912-1028$ & 0.1 & air & 230 & -9.699 & -20.9 & 570 & $\begin{array}{l}\text { Surface thin film of }{ }^{30} \mathrm{Si} \text {, ion } \\
\text { probe profile }\end{array}$ & [69] \\
\hline $\begin{array}{l}\text { B-quarzz } \\
\left(\mathrm{SiO}_{2}\right)\end{array}$ & // c-axis & ${ }^{3} \mathrm{H}_{2} \mathrm{O} "$ & $720-850$ & 0.061 & UB & $100( \pm 1.7)$ & $-10.194( \pm 0.099)$ & -15.1 & 115 & $\begin{array}{l}\text { Exchange with tritiated water } \\
\text { vapor, serial section profiles, } \\
\text { conc. dependent D }\end{array}$ & [145] \\
\hline $\begin{array}{l}\beta \text {-quartz } \\
\left(\mathrm{SiO}_{2}\right)\end{array}$ & $/ / c$-axis & $" \mathrm{H}_{2} \mathrm{O} "$ & 900 & 1500 & NO & n.d. & $\left(\mathrm{D}=10^{-11}\right)$ & & & $\begin{array}{l}\text { Exchange with water/ } \mathrm{D}_{2} \mathrm{O} \text {, } \\
\text { bulk analysis, IR spectra }\end{array}$ & [140] \\
\hline $\begin{array}{l}\text { adularia } \\
\left(\mathrm{Or}_{97.6} \mathrm{Ab}_{1.8} \mathrm{An}_{0.5}\right)\end{array}$ & $\perp(001)$ & ${ }^{18} \mathrm{O}$ & $350-700$ & 100 & UB & $107( \pm 5)$ & $-11.346( \pm 0.301)$ & -16.6 & 178 & $\begin{array}{l}\text { Exchange with }{ }^{18} \mathrm{O} \text {-enriched } \\
\text { water, ion probe profiles }\end{array}$ & {$[66]$} \\
\hline $\begin{array}{l}\text { adularia } \\
\left(\mathrm{Or}_{97.6} \mathrm{Ab}_{1.8} \mathrm{An}_{0.5}\right)\end{array}$ & $\perp(001)$ & ${ }^{18} \mathrm{O}$ & 650 & $\begin{array}{l}5- \\
1500\end{array}$ & $\begin{array}{l}\text { WM } \\
\text { NNO } \\
\end{array}$ & $\begin{array}{l}\text { see paper - D } \\
\text { varies } w / f_{\mathrm{H} 2 \mathrm{O}}\end{array}$ & $\begin{array}{l}\text { independent of } \\
\mathrm{f}_{\mathrm{O} 2}, \mathrm{f}_{\mathrm{H} 2}, \mathrm{a}_{\mathrm{H}+}, \mathrm{P}\end{array}$ & & & $\begin{array}{l}\text { Exchange with }{ }^{18} \mathrm{O} \text {-enriched } \\
\text { water, ion probe profiles }\end{array}$ & [46] \\
\hline $\begin{array}{l}\text { albite (low) } \\
\left(\mathrm{Ab}_{98} \mathrm{Or}_{1.7} \mathrm{An}_{1.2}\right)\end{array}$ & $\perp(001)$ & ${ }^{18} \mathrm{O}$ & $350-700$ & 100 & UB & $89.1( \pm 5.0)$ & $-12.636( \pm 0.019)$ & -17.0 & 146 & $\begin{array}{l}\text { Exchange with }{ }^{18} \mathrm{O} \text {-enriched } \\
\text { water, ion probe profiles }\end{array}$ & [66] \\
\hline $\begin{array}{l}\text { anorthite } \\
\left(\mathrm{An}_{97} \mathrm{Ab}_{3}\right)\end{array}$ & $\perp(001)$ & ${ }^{18} \mathrm{O}$ & $850-1300$ & 0.1 & $\mathrm{O}_{2}$ & $236( \pm 8)$ & $-9.000( \pm 0.349)$ & -20.5 & 553 & $\begin{array}{l}\text { Exchange with }{ }^{18} \mathrm{O}_{2} \text { gas } \\
(+10 \% \mathrm{Ar}) \text {, ion probe profile }\end{array}$ & [42] \\
\hline $\begin{array}{l}\text { anorthite } \\
\left(\mathrm{An}_{94} \mathrm{Ab}_{4}\right) \\
\end{array}$ & $/ /[010]$ & ${ }^{18} \mathrm{O}$ & $1000-1300$ & 0.1 & $\begin{array}{l}(\mathrm{CO}) / \\
\left(\mathrm{CO}_{2}\right) \\
\end{array}$ & $162( \pm 36)$ & $-12.076( \pm 1.337)$ & -20.0 & 439 & $\begin{array}{l}\text { Exchange with }{ }^{18} \mathrm{O} \text {-enriched } \\
\text { gas, jon probe profile }\end{array}$ & [143] \\
\hline $\begin{array}{l}\text { anorthite } \\
\left(\mathrm{An}_{9.3 .3} \mathrm{Ab}_{4.3} \mathrm{Or}_{0.4}\right)\end{array}$ & $\perp(001)$ & ${ }^{18} \mathrm{O}$ & $350-800$ & $\begin{array}{l}100 \\
\text { "wet" }\end{array}$ & UB & $109.6(4.6)$ & $-10.857 \pm(0.021)$ & -16.2 & 172 & $\begin{array}{l}\text { Exchange with }{ }^{18} \mathrm{O} \text {-enriched } \\
\text { water, ion probe profiles }\end{array}$ & [66] \\
\hline $\begin{array}{l}\text { orthoclase } \\
\left(\mathrm{Or}_{94} \mathrm{Ab}_{6}\right)\end{array}$ & powder & ${ }^{22} \mathrm{Na}$ & $500-800$ & 200 & UB & $220.5(+4.6)$ & $-3.050( \pm 0.243)$ & -13.8 & 287 & $\begin{array}{l}\text { Exchange with brine, bulk } \\
\text { analysis, cylindrical model }\end{array}$ & [53] \\
\hline $\begin{array}{l}\text { microcline (max) } \\
\left(\mathrm{Or}_{100}\right)\end{array}$ & powder & ${ }^{22} \mathrm{Na}$ & $600-800$ & 200 & UB & $80( \pm 8)$ & -9.636 & -13.5 & 26 & $\begin{array}{l}\text { Exchange with }{ }^{22} \mathrm{NaCl} \\
\text { solution, sphere model }\end{array}$ & [109] \\
\hline $\begin{array}{l}\text { albite (low) } \\
\left(\mathrm{Ab}_{98} \mathrm{Or}_{1.4} \mathrm{An}_{0.6}\right)\end{array}$ & powder & ${ }^{22} \mathrm{Na}$ & $300-800$ & 200 & UB & $176( \pm 8)$ & $-4.903( \pm 0.814)$ & -13.5 & 218 & $\begin{array}{l}\text { Exchange with }{ }^{22} \mathrm{NaCl} \\
\text { solution, cylindrical model }\end{array}$ & $\begin{array}{l}{[101]} \\
{[163]}\end{array}$ \\
\hline
\end{tabular}




\begin{tabular}{|c|c|c|c|c|c|c|c|c|c|c|c|}
\hline $\begin{array}{c}\text { Mineral, Glass, } \\
\text { or Liquid }\end{array}$ & $\begin{array}{c}\text { Orienta- } \\
\text { tion }\end{array}$ & $\begin{array}{c}\text { Diffusing } \\
\text { Component }\end{array}$ & $\begin{array}{r}\text { Temperature } \\
\text { Range }\left({ }^{\circ} \mathrm{C}\right)\end{array}$ & $\begin{array}{c}\mathrm{P} \\
(\mathrm{MPa}) \\
\end{array}$ & $\begin{array}{c}\mathrm{O}_{2} \\
(\mathrm{MPa}) \\
\end{array}$ & $\begin{array}{c}\Delta \mathrm{E}_{\mathrm{a}} \\
(\mathrm{kJ} / \mathrm{mole})\end{array}$ & $\begin{array}{c}\log D_{0} \text { (or D) } \\
\left(\mathrm{m}^{2} / \mathrm{s}\right)\end{array}$ & $\begin{array}{l}\log \mathrm{D} \\
800^{\circ} \mathrm{C} \\
\end{array}$ & $\begin{array}{l}{ }^{\prime \prime} \mathrm{T}_{\mathrm{c}} " \\
\left({ }^{\circ} \mathrm{C}\right) \\
\end{array}$ & Experiment/Comments & Ref. \\
\hline $\begin{array}{l}\text { orthoclase } \\
\left(\mathrm{Or}_{94} \mathrm{Ab}_{6}\right)\end{array}$ & powder & ${ }^{40} \mathrm{Ar}$ & $500-800$ & 200 & UB & $180.3( \pm 4.6)$ & $-5.854( \pm 0.259)$ & -14.6 & 256 & $\begin{array}{l}\text { Ar loss into brine, bulk } \\
\text { analysis, spherical model }\end{array}$ & {$[52]$} \\
\hline $\begin{array}{l}\text { microcline (max) } \\
\left(\mathrm{Or}_{100}\right)\end{array}$ & powder & ${ }^{40} \mathrm{~K}$ & $600-800$ & 200 & UB & $293( \pm 8)$ & -1.874 & -16.1 & 428 & $\begin{array}{l}\text { Exchange with }{ }^{40} \mathrm{KCl} \\
\text { solution, sphere model }\end{array}$ & [109] \\
\hline $\begin{array}{l}\text { albite (low) } \\
\left(\mathrm{Ab}_{98} \mathrm{Or}_{1.4} \mathrm{An}_{0.6}\right)\end{array}$ & powder & ${ }^{40} \mathrm{~K}$ & $600-800$ & 200 & UB & $172( \pm 25)$ & $-8.125( \pm 0.337)$ & -16.5 & 301 & $\begin{array}{l}\text { Exchange with }{ }^{40} \mathrm{KCl} \\
\text { solution, cylindrical model }\end{array}$ & $\begin{array}{l}{[101]} \\
{[163]}\end{array}$ \\
\hline $\begin{array}{l}\text { orthoclase } \\
\left(\mathrm{Or}_{94} \mathrm{Ab}_{6}\right)\end{array}$ & powder & ${ }^{41} \mathrm{~K}$ & $600-800$ & 200 & UB & $285.4( \pm 3.8)$ & $-2.793( \pm 0.190)$ & -16.7 & 439 & $\begin{array}{l}\text { Exchange with brine, bulk } \\
\text { analysis, cylindrical model }\end{array}$ & [53] \\
\hline $\begin{array}{l}\text { orthoclase } \\
\left(\mathrm{Or}_{94} \mathrm{Ab}_{6}\right)\end{array}$ & $/ / c$-axis & ${ }^{87} \mathrm{Rb}$ & $625-800$ & 100 & UB & $339( \pm 33)$ & $-2.000( \pm 1.800)$ & -18.5 & 541 & $\begin{array}{l}\text { Exchange w/Rb-Sr-enriched } \\
\text { water, ion probe profiles }\end{array}$ & {$[63]$} \\
\hline $\begin{array}{l}\text { albite } \\
\left(\mathrm{Ab} b_{98}\right)\end{array}$ & $\perp(001)$ & ${ }^{86} \mathrm{Sr}$ & $570-1080$ & 0.1 & air & 272 & -4.509 & -17.7 & 465 & $\begin{array}{l}\text { Thin film of }{ }^{86} \mathrm{Sr} \text {, ion probe } \\
\text { profile, other plag in progress }\end{array}$ & {$[64]$} \\
\hline $\begin{array}{l}\text { orthoclase } \\
\left(\mathrm{Or}_{94} \mathrm{Ab}_{6}\right)\end{array}$ & //c-axis & ${ }^{84} \mathrm{Sr}$ or ${ }^{86} \mathrm{Sr}$ & $625-900$ & 100 & UB & $167( \pm 17)$ & $-11.000( \pm 0.900)$ & -19.1 & 405 & $\begin{array}{l}\text { Exchange w/Rb-Sr-cnriched } \\
\text { water, ion probe profiles }\end{array}$ & [63] \\
\hline $\begin{array}{l}\text { orthoclase } \\
\left(\mathrm{Or}_{93}\right)\end{array}$ & $\perp(001)$ & $\begin{array}{l}\text { SrAl-KSi } \\
\text { interdiffusion }\end{array}$ & $725-1075$ & 0.1 & air & $284.1( \pm 6.7)$ & $-6.224( \pm 0.302)$ & -20.1 & 568 & $\begin{array}{l}\text { Exchange w/Sr-Al-Si-O } \\
\text { powder, Rutherford Back. Spec }\end{array}$ & {$[24]$} \\
\hline $\begin{array}{l}\text { anothoclase } \\
\left(\mathrm{Ab}_{68} \mathrm{Or}_{27} \mathrm{An}_{5}\right)\end{array}$ & $\perp(001)$ & $\begin{array}{l}\text { SrAl-KSi } \\
\text { interdiffusion }\end{array}$ & $725-1075$ & 0.1 & air & $373.7( \pm 19.2)$ & $-1.648( \pm 0.913)$ & -19.8 & 609 & $\begin{array}{l}\text { Exchange w/Sr-Al-Si-O } \\
\text { powder, Rutherford Back. Spec }\end{array}$ & [24] \\
\hline $\begin{array}{l}\text { anothoclase } \\
\left(\mathrm{Ab}_{68} \mathrm{Or}_{27} \mathrm{An}_{5}\right)\end{array}$ & $\perp(010)$ & $\begin{array}{l}\text { SrAl-KSi } \\
\text { interdiffusion }\end{array}$ & $725-1075$ & 0.1 & air & $372.8( \pm 20.1)$ & $-2.346( \pm 0.951)$ & -20.5 & 634 & $\begin{array}{l}\text { Exchange w/Sr-Al-Si-O } \\
\text { powder, Rutherford Back. Spcc }\end{array}$ & [24] \\
\hline $\begin{array}{l}\text { anorthite } \\
\left(\mathrm{Ab}_{6} \mathrm{An}_{93}\right) \\
\end{array}$ & $\perp(010)$ & $\begin{array}{l}\text { Sr-Ca } \\
\text { interdiffusion }\end{array}$ & $725-1075$ & 0.1 & air & $329.7( \pm 22.6)$ & $-5.415( \pm 1.037)$ & -21.5 & 660 & $\begin{array}{l}\text { Exchange w/Sr-Al-Si-O } \\
\text { powder, Rutherford Back. Spec }\end{array}$ & [24] \\
\hline $\begin{array}{l}\text { alhite (low) } \\
\left(\mathrm{Ab}_{98} \mathrm{Or}_{1.4} \mathrm{An}_{0.6}\right)\end{array}$ & $/ / \mathrm{c}$-axis & ${ }^{84} \mathrm{Sr}$ & $640-800$ & 100 & UB & $247( \pm 25)$ & $-5.600( \pm 1.300)$ & -17.6 & 437 & $\begin{array}{l}\text { Exchange w/Rb-Sr-enriched } \\
\text { water, ion probe profiles }\end{array}$ & [63] \\
\hline $\begin{array}{l}\text { adularia }\left(\mathrm{Or}_{89.6}\right)- \\
\text { albite }\left(\mathrm{Ab}_{98.6}\right)\end{array}$ & $\begin{array}{l}\perp(001) \\
\text { (couple) }\end{array}$ & $\begin{array}{l}\mathrm{K}-\mathrm{Na} \\
\text { interdiffusion }\end{array}$ & $900-1000$ & 1500 & UB & n.d. & $\begin{array}{l}\left(\mathrm{D}=10^{-17} \text { to }\right. \\
\left.10^{-15}\right)\end{array}$ & & & $\begin{array}{l}\text { Microprobe profile, composi- } \\
\text { tion dependence, anisotropy }\end{array}$ & [25] \\
\hline $\begin{array}{l}\text { albite }\left(\mathrm{Ab}_{92}\right) \\
\text { exsolved }\left(\mathrm{An}_{026}\right)\end{array}$ & $\begin{array}{l}\perp(0 \overline{4} 1) \\
\text { (couple) }\end{array}$ & $\begin{array}{l}\text { CaAl-NaSi } \\
\text { interdiffusion }\end{array}$ & $900-1050$ & $\begin{array}{l}1500 \\
\text { "wet" }\end{array}$ & $\mathrm{MH}$ & $303( \pm 35)$ & $-7.523( \pm 0.300)$ & -22.3 & 691 & $\begin{array}{l}\text { Average D from lamellar } \\
\text { homogenization experiments }\end{array}$ & [114] \\
\hline $\begin{array}{l}\text { bytownite }\left(A n_{80}\right) \\
\text { exsolved }\left(A n_{70 / 90}\right)\end{array}$ & $\begin{array}{l}\perp(03 \overline{1}) \\
\text { (couple) }\end{array}$ & $\begin{array}{l}\mathrm{CaAl}-\mathrm{NaSi} \\
\text { interdiffusion } \\
\end{array}$ & $1100-1400$ & 0.1 & air & $516.3( \pm 19)$ & $-2.959( \pm 0.662)$ & -28.1 & 1011 & $\begin{array}{l}\text { Average D from lamellar } \\
\text { homogenization experiments }\end{array}$ & [74] \\
\hline $\begin{array}{l}\text { bytownite }\left(\mathrm{An}_{80}\right) \\
\text { exsolved }\left(\mathrm{An}_{70 / 90}\right)\end{array}$ & $\begin{array}{l}\perp(03 \overline{1}) \\
\text { (couple) }\end{array}$ & $\begin{array}{l}\mathrm{CaAl}-\mathrm{NaSi} \\
\text { interdiffusion } \\
\end{array}$ & $\begin{array}{l}900-975 \\
1000-1050 \\
\end{array}$ & $\begin{array}{l}1500 \\
\text { "wet" }\end{array}$ & $\mathrm{MH}$ & $\begin{array}{l}-317( \pm 35) \\
-103 \\
\end{array}$ & $\begin{array}{l}-4.959( \pm 0.300) \\
-15.398 \\
\end{array}$ & $\begin{array}{l}-20.4 \\
-20.4 \\
\end{array}$ & $\begin{array}{l}604 \\
350 \\
\end{array}$ & $\begin{array}{l}\text { Average D's from lamellar } \\
\text { homogenization experiments }\end{array}$ & {$[114]$} \\
\hline K-feldspar $\left(\mathrm{Or}_{93}\right)$ & $\perp(010)$ & $\begin{array}{l}\mathrm{Pb}-? \\
\text { interdiffusion }\end{array}$ & $750-1050$ & 0.1 & UB & $301.7( \pm 11.3)$ & $-6.000(+0.519)$ & -20.7 & 609 & $\begin{array}{l}\text { Exchange with } \mathrm{PbS} \text { powder, } \\
\text { Rutherford backscattering }\end{array}$ & $\lceil 21\rceil$ \\
\hline K-feldspar $\left(\mathrm{Or}_{93}\right)$ & $\perp(001)$ & $\begin{array}{l}\mathrm{Pb}-? \\
\text { interdiffusion }\end{array}$ & $750-1050$ & 0.1 & UB & $306.7( \pm 26.8)$ & $-4.886(11.176)$ & -19.8 & 573 & $\begin{array}{l}\text { Exchange with } \mathrm{PbS} \text { powder, } \\
\text { Rutherford backscattering }\end{array}$ & [21] \\
\hline Oligoclase $\left(\mathrm{An}_{23}\right)$ & $\perp(010)$ & $\begin{array}{l}\mathrm{Pb}-? \\
\text { interdiffusion }\end{array}$ & $750-1050$ & 0.1 & UB & $364.5( \pm 12.1)$ & $-2.921( \pm 0.540)$ & -20.7 & 638 & $\begin{array}{l}\text { Exchange with } \mathrm{PbS} \text { powder, } \\
\text { Rutherford backscattering }\end{array}$ & [21] \\
\hline Oligoclase $\left(\mathrm{An}_{23}\right)$ & $\perp(001)$ & $\begin{array}{l}\mathrm{Pb}-\text { ? } \\
\text { interdiffusion }\end{array}$ & $750-1050$ & 0.1 & UB & $226.0( \pm 9.2)$ & $-8.387( \pm 0.415)$ & -19.4 & 489 & $\begin{array}{l}\text { Exchange with } \mathrm{PbS} \text { powder, } \\
\text { Rutherford backscattering }\end{array}$ & [21] \\
\hline
\end{tabular}




\begin{tabular}{|c|c|c|c|c|c|c|c|c|c|c|c|}
\hline $\begin{array}{l}\text { Mineral, Glass, } \\
\text { or Liquid } \\
\end{array}$ & $\begin{array}{c}\text { Orienta- } \\
\text { tion }\end{array}$ & $\begin{array}{c}\text { Diffusing } \\
\text { Component } \\
\end{array}$ & \begin{tabular}{|c|} 
Temperature \\
Range $\left({ }^{\circ} \mathrm{C}\right)$ \\
\end{tabular} & $\begin{array}{c}\mathrm{P} \\
(\mathrm{MPa}) \\
\end{array}$ & $\begin{array}{c}\mathrm{O}_{2} \\
(\mathrm{MPa}) \\
\end{array}$ & $\begin{array}{c}\Delta \mathrm{E}_{\mathrm{a}} \\
(\mathrm{kJ} / \mathrm{mole}) \\
\end{array}$ & $\begin{array}{c}\log \mathrm{D}_{\mathrm{o}}(\text { or } \mathrm{D}) \\
\left(\mathrm{m}^{2} / \mathrm{s}\right) \\
\end{array}$ & $\begin{array}{l}\log \mathrm{D} \\
800^{\circ} \mathrm{C} \\
\end{array}$ & $\begin{array}{l}" \mathrm{~T}_{\mathrm{c}} " \\
\left({ }^{\circ} \mathrm{C}\right) \\
\end{array}$ & Experiment/Comments & Ref. \\
\hline nepheline & powder & ${ }^{18} \mathrm{O}$ & $1000-1300$ & 0.1 & $\mathrm{CO}_{2}$ & $104.6( \pm 10.5)$ & -12.229 & -17.3 & 199 & $\begin{array}{l}\text { Exchange with } \mathrm{CO}_{2} \text {, spherical } \\
\text { model, bulk analysis }\end{array}$ & [27] \\
\hline $\begin{array}{l}\text { biotite } \\
\text { (see paper for } \\
\text { comp.) }\end{array}$ & powder & ${ }^{18} \mathrm{O}$ & $500-800$ & $\begin{array}{l}100 \\
\text { "wet" }\end{array}$ & $\begin{array}{l}\text { UB/ } \\
\text { NO }\end{array}$ & $142(+8)$ & -9.041 & -16.0 & 233 & $\begin{array}{l}\text { Exchange with }{ }^{18} \mathrm{O} \text {-enriched } \\
\text { water, bulk analysis, cylin- } \\
\text { drical model, ion probe too }\end{array}$ & [54] \\
\hline $\begin{array}{l}\text { phlogopite } \\
\text { (see paper for } \\
\text { comp.) }\end{array}$ & powder & ${ }^{18} \mathrm{O}$ & $600-900$ & $\begin{array}{l}100 \\
\text { "wet" }\end{array}$ & $\begin{array}{l}\text { UB/ } \\
\text { NO }\end{array}$ & $176( \pm 13)$ & -7.854 & -16.4 & 305 & $\begin{array}{l}\text { Exchange with }{ }^{18} \mathrm{O} \text {-enriched } \\
\text { water, bulk analysis, cylin- } \\
\text { drical model, ion probe too }\end{array}$ & [54] \\
\hline $\begin{array}{l}\text { phlogopite } \\
\text { (Ann } 4 \text { ) (see paper } \\
\text { for comp.) } \\
\end{array}$ & powder & ${ }^{40} \mathrm{Ar}$ & $600-900$ & $\begin{array}{l}200 \\
1500 \\
\text { "wet" }\end{array}$ & $\begin{array}{l}\text { UB/ } \\
\text { NO }\end{array}$ & $242( \pm 11)$ & $-4.125( \pm 0.514)$ & -15.9 & 373 & $\begin{array}{l}\text { Degassing into water, bulk } \\
\text { analysis, cylindrical model, } \\
\left(\Delta \mathrm{V}_{a} \cong 0 \mathrm{~m}^{3} / \text { mole }\right)\end{array}$ & $\begin{array}{l}{[62]} \\
{[67]}\end{array}$ \\
\hline $\begin{array}{l}\text { biotitc }\left(A_{56} n_{56}\right) \\
\text { (see paper for } \\
\text { comp. reference) } \\
\end{array}$ & powder & ${ }^{40} \mathrm{Ar}$ & $600-750$ & $\begin{array}{l}100 \\
1400 \\
\text { "wet" }\end{array}$ & $\begin{array}{l}\text { GM, } \\
\text { QFM }\end{array}$ & $197( \pm 9)$ & $-5.114( \pm 0.614)$ & -14.7 & 281 & $\begin{array}{l}\text { Degassing into water, bulk } \\
\text { analysis, cylindrical model } \\
\left(\Delta \mathrm{V}_{\mathrm{a}}=1.4 \times 10^{-5} \mathrm{~m}^{3} / \mathrm{mole}\right)\end{array}$ & [77] \\
\hline biotite & powder & ${ }^{41} \mathrm{~K}$ & $450-700$ & $\begin{array}{l}200 \\
\text { "wet" }\end{array}$ & $\begin{array}{l}\text { UB/ } \\
\text { NO }\end{array}$ & 88 & & & & $\begin{array}{l}\text { Exchange with }{ }^{41} \mathrm{KCl} \text { solu- } \\
\text { tion, bulk analysis \& ion } \\
\text { probe, cylindrical model }\end{array}$ & $\begin{array}{l}{[87]} \\
{[88]}\end{array}$ \\
\hline $\begin{array}{l}\text { chlorite } \\
\text { (sheridanitc) } \\
\text { (see paper) }\end{array}$ & powder & ${ }^{2} \mathrm{H}$ & $500-700$ & $\begin{array}{l}200 \& \\
500\end{array}$ & $\begin{array}{l}\text { UB/ } \\
\text { NO }\end{array}$ & 171.7 & -5.21 & -13.6 & 214 & $\begin{array}{l}\text { Enchange with }{ }^{2} \mathrm{H} \text {-selected } \\
\text { water, bulk analysis, } \\
\text { cylindrical model }\end{array}$ & {$[72]$} \\
\hline mucovite & powder & ${ }^{2} \mathrm{H}$ & $450-750$ & $\begin{array}{l}200 \& \\
400\end{array}$ & UB & 121.3 & -7.98 & -13.9 & 133 & $\begin{array}{l}\text { Enchange with }{ }^{2} \mathrm{H} \text {-selected } \\
\text { water, bulk analysis, } \\
\text { cylindrical model }\end{array}$ & {$[70]$} \\
\hline $\begin{array}{l}\text { muscovite (see } \\
\text { paper for comp.) }\end{array}$ & powder & ${ }^{18} \mathrm{O}$ & $512-700$ & $\begin{array}{l}100 \\
\text { "wet" }\end{array}$ & $\begin{array}{l}\text { UB/ } \\
\text { NO }\end{array}$ & $163( \pm 21)$ & -8.114 & -16.1 & 273 & $\begin{array}{l}\text { Exchange with }{ }^{18} \mathrm{O} \text {-enriched } \\
\text { water, bulk analysis, cylin- } \\
\text { drical model, ion probe too }\end{array}$ & [54] \\
\hline $\begin{array}{l}\text { tremolite (see } \\
\text { paper for comp.) }\end{array}$ & //c-axis & ${ }^{18} \mathrm{O}$ & $650-800$ & $\begin{array}{l}100 \\
\text { "wet" }\end{array}$ & $\begin{array}{l}\text { UB/ } \\
\text { NO }\end{array}$ & $163( \pm 21)$ & $-11.699( \pm 1.204)$ & -19.6 & 424 & $\begin{array}{l}\text { Exchange with }{ }^{18} \mathrm{O} \text {-enriched } \\
\text { water, ion probe profiles }\end{array}$ & [44] \\
\hline $\begin{array}{l}\text { hornblende (see } \\
\text { paper for comp.) }\end{array}$ & $/ / \mathrm{c}$-axis & ${ }^{18} \mathrm{O}$ & $650-800$ & $\begin{array}{l}100,20 \\
2000 \\
\text { "wet" } \\
\end{array}$ & $\begin{array}{l}\text { UB/ } \\
\text { NO }\end{array}$ & $172( \pm 25)$ & $-11.000( \pm 1.322)$ & -19.4 & 421 & $\begin{array}{l}\text { Exchange with }{ }^{18} \mathrm{O} \text {-enriched } \\
\text { water, ion probe profiles, } \\
\text { anisotropy, pressure depend. }\end{array}$ & [44] \\
\hline $\begin{array}{l}\text { richterite (see } \\
\text { paper for comp.) }\end{array}$ & $\not / c$-axis & ${ }^{18} \mathrm{O}$ & $650-800$ & $\begin{array}{l}100 \\
\text { "wet" }\end{array}$ & $\begin{array}{l}\text { UB/ } \\
\mathrm{NO}\end{array}$ & $239( \pm 8)$ & $-7.523( \pm 0.452)$ & -19.1 & 490 & $\begin{array}{l}\text { Exchange with }{ }^{18} \mathrm{O} \text {-enriched } \\
\text { water, ion probe profiles }\end{array}$ & [44] \\
\hline $\begin{array}{l}\text { hornblende (see } \\
\text { paper for comp.) }\end{array}$ & & ${ }^{40} \mathrm{Ar}$ & $750-900$ & $\begin{array}{l}100 \\
\text { "wet" }\end{array}$ & $\begin{array}{l}\text { UB or } \\
\text { NO }\end{array}$ & $268.2( \pm 7.1)$ & $-5.620( \pm 0.506)$ & -18.7 & 497 & $\begin{array}{l}\text { Degassing into water, bulk } \\
\text { analysis, spherical model }\end{array}$ & [76] \\
\hline tremolite & $/ / c$-axis & $\mathrm{Sr}$ & 800 & $\begin{array}{l}200 \\
\text { "wet" }\end{array}$ & $\begin{array}{l}\text { UB/ } \\
\text { NO }\end{array}$ & & $\mathrm{D}=1 \times 10^{-21}$ & & & $\begin{array}{l}\text { Thin film solution, ion probe } \\
\text { profile }\end{array}$ & [9] \\
\hline
\end{tabular}




\begin{tabular}{|c|c|c|c|c|c|c|c|c|c|c|c|}
\hline $\begin{array}{c}\text { Mineral, Glass, } \\
\text { or Liquid }\end{array}$ & $\begin{array}{c}\text { Orienta- } \\
\text { tion }\end{array}$ & $\begin{array}{l}\text { Diffusing } \\
\text { Component } \\
\end{array}$ & \begin{tabular}{|r|} 
Temperature \\
Range $\left({ }^{\circ} \mathrm{C}\right)$ \\
\end{tabular} & $\begin{array}{c}\mathrm{P} \\
(\mathrm{MPa}) \\
\end{array}$ & $\begin{array}{c}\mathrm{O}_{2} \\
(\mathrm{MPa}) \\
\end{array}$ & $\begin{array}{c}\wedge \mathrm{F}_{\mathrm{a}} \\
(\mathrm{kJ} / \mathrm{mole}) \\
\end{array}$ & $\begin{array}{c}\log D_{0}(\mathrm{or} D) \\
\left(\mathrm{m}^{2} / \mathrm{s}\right)\end{array}$ & $\begin{array}{l}\log \mathrm{D} \\
800^{\circ} \mathrm{C} \\
\end{array}$ & $\begin{array}{l}" \mathrm{~T}_{\mathrm{c}} " \\
\left({ }^{\circ} \mathrm{C}\right) \\
\end{array}$ & Experiment/Comments & Ref. \\
\hline orthopyroxene & powder & $\begin{array}{l}\text { Fe-Mg } \\
\text { interdiffusion }\end{array}$ & $600-800$ & 0.1 & $\begin{array}{l}-12.2- \\
-17.9\end{array}$ & 233 & -9.432 & -20.8 & 564 & $\begin{array}{l}\text { Calculated from disordering } \\
\text { experiments [7] }\end{array}$ & [60] \\
\hline $\begin{array}{l}\text { diopside } \\
\left(\mathrm{Ca}_{92} \mathrm{Mg}_{9.98} \mathrm{Fe}_{.10}\right)\end{array}$ & powder & $\begin{array}{l}\text { He "apparent } \\
\text { diffusivity" }\end{array}$ & $700-1400$ & 0.0 & UB & $290( \pm 40)$ & $-1.9( \pm 1.2)$ & -16.0 & 422 & $\begin{array}{l}\text { Degassing experiment, } \\
\text { spherical model, fractures! }\end{array}$ & [151] \\
\hline $\begin{array}{l}\text { diopside } \\
\text { (synthetic) }\end{array}$ & powder & ${ }^{18} \mathrm{O}$ & $1150-1350$ & 0.1 & $\mathrm{CO}_{2}$ & $59( \pm 13)$ & $-14.886( \pm 0.520)$ & -17.7 & 71 & $\begin{array}{l}\text { Exchange with } \mathrm{CO}_{2} \text {, spherical } \\
\text { model, bulk analysis }\end{array}$ & [130] \\
\hline $\begin{array}{l}\text { diopside }\left(W_{0} o_{50.6}\right. \\
\left.E_{48.3} F_{5.1 .1}\right)\end{array}$ & $/ / \mathrm{c}$-axis & ${ }^{18} \mathrm{O}$ & $1100-1250$ & 0.1 & NNO & $-457( \pm 26)$ & $-3.367( \pm 0.934)$ & -25.6 & 888 & $\begin{array}{l}\text { Exchange with }{ }^{18} \mathrm{O} \text {-enriched } \\
\text { gas, ion probe profiles }\end{array}$ & [143] \\
\hline $\begin{array}{l}\text { diopside ("essen- } \\
\text { tially pure") }\end{array}$ & $/ /$ c-axis & ${ }^{18} \mathrm{O}$ & $700-1250$ & $\begin{array}{l}100 \\
\text { "wet" }\end{array}$ & UB & $226( \pm 21)$ & -9.824 & -20.8 & 562 & $\begin{array}{l}\text { Exchange with }{ }^{18} \mathrm{O} \text {-enriched } \\
\text { water, ion probe profiles }\end{array}$ & [43] \\
\hline $\begin{array}{l}\text { diopside ("essen- } \\
\text { lially pure") }\end{array}$ & $\perp c$-axis & ${ }^{18} \mathrm{O}$ & $700-1250$ & $\begin{array}{l}100 \\
\text { "wet" }\end{array}$ & UB & 226 & -11.553 & -22.6 & 671 & $\begin{array}{l}\text { Exchange with }{ }^{18} \mathrm{O} \text {-enriched } \\
\text { water, ion probe profiles }\end{array}$ & [43] \\
\hline $\begin{array}{l}\text { diopside }\left(\mathrm{Na}_{04}\right. \\
\mathrm{Ca}_{96} \mathrm{Mg}_{.96} \mathrm{Fe}_{05} \\
\left.\mathrm{Al}_{06} \mathrm{~S}_{1.96} \mathrm{O}_{6}\right)\end{array}$ & $/ / c$-axis & $\begin{array}{l}\text { Al-? } \\
\text { interdiffusion }\end{array}$ & 1180 & 0.1 & $\begin{array}{l}\mathrm{P}\left(\mathrm{O}_{2}\right) \\
=10^{-14}\end{array}$ & n.d. & $\begin{array}{l}(\mathrm{D}=3.2( \pm 0.7) \\
\left.\times 10^{-21}\right)\end{array}$ & & & $\begin{array}{l}\text { Thin film of amorphous } \\
\mathrm{CaAl}_{2} \mathrm{SiO}_{6},{ }^{27} \mathrm{Al}(\mathrm{p}, \gamma)^{28} \mathrm{Si} \\
\text { nuclear reaction profile }\end{array}$ & [144] \\
\hline $\begin{array}{l}\text { diopside } \\
\text { (synthetic) } \\
\left(\mathrm{CaMgSi}_{2} \mathrm{O}_{6}\right)\end{array}$ & $/ / c$-axis & $\mathrm{Sr}$ & $1100-1250$ & 2000 & UB & $607(+33)$ & 2.940 & -26.6 & 911 & $\begin{array}{l}\text { Thin film, sectioning, ion } \\
\text { probe, Rutherford backscatter, } \\
\text { anisotropy, pressure depend. }\end{array}$ & [149] \\
\hline $\begin{array}{l}\text { diopside } \\
\left(\text { Wo }_{99} \mathrm{En}_{99} \mathrm{Fs}_{02} \text { ) }\right.\end{array}$ & $/ / c$-axis & ${ }^{85} \mathrm{Sr}$ & $1100-1300$ & 0.1 & $\begin{array}{l}\mathrm{UB} / \\
\mathrm{N}_{2}\end{array}$ & 406 & -2.268 & -22.0 & 710 & $\begin{array}{l}\text { Thin film, sectioning by } \\
\text { grinding, scintillation counts }\end{array}$ & {$[149]$} \\
\hline $\begin{array}{l}\text { diopside } \\
\text { (synthetic) } \\
\left(\mathrm{CaMgSi}_{2} \mathrm{O}_{6}\right) \\
\end{array}$ & $/ /$ c-axis & $\mathrm{Sm}$ & $1100-1250$ & $\begin{array}{l}0.1 \text { to } \\
2000\end{array}$ & $\mathrm{UB}$ & $590(+96)$ & 2.146 & .26 .6 & 913 & $\begin{array}{l}\text { Thin film, sectioning, ion } \\
\text { probe, Rutherford backscatter, } \\
\text { anisotropy, pressure depend. }\end{array}$ & [149] \\
\hline $\begin{array}{l}\text { clinopyroxene } \\
\left(\mathrm{Na}_{.1} \mathrm{Ca}_{.53} \mathrm{Mg}_{1.1}\right. \\
\left.\mathrm{Fc}_{.17} \mathrm{Al}_{.1} \mathrm{Si}_{2.0} \mathrm{O}_{6}\right)\end{array}$ & $\perp(001)$ & $\begin{array}{l}\mathrm{Ca}-(\mathrm{Mg}, \mathrm{Fe}) \\
\text { interdiffusion }\end{array}$ & $1150-1250$ & 2500 & $\begin{array}{l}\text { UB/ } \\
\text { GrPC }\end{array}$ & $\begin{array}{l}360.87 \\
( \pm 190)\end{array}$ & -6.410 & -24.0 & 801 & $\begin{array}{l}\text { Average } D \text { from lamellar } \\
\text { homogenization experiments }\end{array}$ & [12] \\
\hline $\begin{array}{l}\text { wollastonite (syn- } \\
\text { thetic? } \alpha-\mathrm{CaSiO}_{3} \text { ) }\end{array}$ & $\begin{array}{l}\text { sintered } \\
\text { powder }\end{array}$ & ${ }^{45} \mathrm{Ca}$ & $900-1300 ?$ & 0.006 & UB & 469 & 0.845 & -22.0 & 721 & $\begin{array}{l}\text { Thin film, autoradiography } \\
\text { profiles? }\end{array}$ & $\begin{array}{l}{[110]} \\
{[111]}\end{array}$ \\
\hline $\begin{array}{l}\text { åkermanite (syn) } \\
\left(\mathrm{Ca}_{2} \mathrm{MgSi}_{2} \mathrm{O}_{7}\right)\end{array}$ & $/ / \mathrm{c}$-axis & ${ }^{18} \mathrm{O}$ & $1000-1300$ & 0.1 & $\mathrm{CO}_{2}$ & $215( \pm 51)$ & -9.026 & -19.5 & 483 & $\begin{array}{l}\text { Exchange with } \mathrm{C}^{18} \mathrm{O}_{2} \\
\text { atmosphere, ion probe profile }\end{array}$ & {$[165]$} \\
\hline $\begin{array}{l}\text { åkermanite (syn) } \\
\left(\mathrm{Ca}_{2} \mathrm{MgSi}_{2} \mathrm{O}_{7}\right)\end{array}$ & $\perp c$-axis & ${ }^{18} \mathrm{O}$ & $800-1300$ & 0.1 & $\begin{array}{l}(\mathrm{CO}) / \\
\left(\mathrm{CO}_{2}\right)\end{array}$ & $278( \pm 33)$ & $-6.328( \pm 1.282)$ & -19.9 & 555 & $\begin{array}{l}\text { Exchange with }{ }^{18} \mathrm{O} \text {-enriched } \\
\text { gass, ion probe profile }\end{array}$ & [143] \\
\hline $\begin{array}{l}\text { åkermanite (syn) } \\
\left(\mathrm{Ca}_{2} \mathrm{MgSi}_{2} \mathrm{O}_{7}\right)\end{array}$ & $/ / c$-axis & ${ }^{45} \mathrm{Ca}$ & $1100-1300$ & 0.1 & $\mathrm{~N}_{2}$ & 410 & -0.301 & -20.3 & 639 & $\begin{array}{l}\text { Thin film, sectioning by } \\
\text { grinding, grindings counted }\end{array}$ & [127] \\
\hline $\begin{array}{l}\text { åkermanite (syn) } \\
\left(\mathrm{Ca}_{2} \mathrm{MgSi}_{2} \mathrm{O}_{7}\right)\end{array}$ & //c-axis & ${ }^{54} \mathrm{Mn}$ & $1100-1300$ & 0.1 & $\mathrm{~N}_{2}$ & 300 & -4.569 & -19.2 & 541 & $\begin{array}{l}\text { Thin film, sectioning by } \\
\text { grinding, grindings counted }\end{array}$ & [127] \\
\hline $\begin{array}{l}\text { åkermanite (syn) } \\
\left(\mathrm{Ca}_{2} \mathrm{MgSi}_{2} \mathrm{O}_{7}\right)\end{array}$ & $/ /$ c-axis & ${ }^{59} \mathrm{Fe}$ & $1100-1300$ & 0.1 & $\mathrm{~N}_{2}$ & 230 & -7.377 & -18.6 & 457 & $\begin{array}{l}\text { Thin film, sectioning by } \\
\text { grinding, grindings counted }\end{array}$ & [127] \\
\hline $\begin{array}{l}\text { åkermanite (syn) } \\
\left(\mathrm{Ca}_{2} \mathrm{MgSi}_{2} \mathrm{O}_{7}\right)\end{array}$ & $/ / \mathrm{c}$-axis & ${ }^{60} \mathrm{Co}$ & $1100-1300$ & 0.1 & $\mathrm{~N}_{2}$ & 230 & -7.770 & -19.0 & 474 & $\begin{array}{l}\text { Thin film, sectioning by } \\
\text { grinding, grindings counted }\end{array}$ & [127] \\
\hline
\end{tabular}




\begin{tabular}{|c|c|c|c|c|c|c|c|c|c|c|c|}
\hline $\begin{array}{c}\text { Mineral, Glass, } \\
\text { or Liquid } \\
\end{array}$ & $\begin{array}{c}\text { Orienta- } \\
\text { tion }\end{array}$ & $\begin{array}{l}\text { Diffusing } \\
\text { Component } \\
\end{array}$ & $\begin{array}{r}\text { Temperature } \\
\text { Range }\left({ }^{\circ} \mathrm{C}\right) \\
\end{array}$ & $\begin{array}{c}\mathrm{P} \\
(\mathrm{MPa}) \\
\end{array}$ & $\begin{array}{c}\mathrm{O}_{2} \\
(\mathrm{MPa})\end{array}$ & $\begin{array}{c}\Delta \mathrm{E}_{\mathrm{a}} \\
(\mathrm{kJ} / \mathrm{mole})\end{array}$ & $\begin{array}{c}\log D_{0}(\text { or } D) \\
\left(\mathrm{m}^{2} / \mathrm{s}\right) \\
\end{array}$ & $\begin{array}{l}\log \mathrm{D} \\
800^{\circ} \mathrm{C} \\
\end{array}$ & $\begin{array}{l}" \mathrm{~T}_{\mathrm{c}} " \\
\left({ }^{\circ} \mathrm{C}\right) \\
\end{array}$ & Experiment/Comments & Ref. \\
\hline $\begin{array}{l}\text { åkermanite (syn) } \\
\left(\mathrm{Ca}_{2} \mathrm{MgSi}_{2} \mathrm{O}_{7}\right)\end{array}$ & $/ / c$-axis & ${ }^{63} \mathrm{Ni}$ & $1100-1300$ & 0.1 & $\mathrm{~N}_{2}$ & 200 & -8.301 & -18.0 & 400 & $\begin{array}{l}\text { Thin film, sectioning by } \\
\text { grinding, grindings counted }\end{array}$ & [127] \\
\hline $\begin{array}{l}\text { åkermanite (syn) } \\
\left(\mathrm{Ca}_{2} \mathrm{MgSi}_{2} \mathrm{O}_{7}\right)\end{array}$ & $/ / \mathrm{c}$-axis & ${ }^{85} \mathrm{Sr}$ & $1100-1300$ & 0.1 & $\mathrm{~N}_{2}$ & 380 & -1.745 & -20.2 & 627 & $\begin{array}{l}\text { Thin film, sectioning by } \\
\text { grinding, grindings counted }\end{array}$ & [127] \\
\hline $\begin{array}{l}\text { åkermanite (syn) } \\
\left(\mathrm{Ca}_{2} \mathrm{MgSi}_{2} \mathrm{O}_{7}\right)\end{array}$ & $/ / c$-axis & ${ }^{133} \mathrm{Ba}$ & $1100-1300$ & 0.1 & $\mathrm{~N}_{2}$ & 290 & -4.854 & -19.0 & 526 & $\begin{array}{l}\text { Thin film, sectioning by } \\
\text { grinding, grindings counted }\end{array}$ & $\lceil 127\rceil$ \\
\hline $\begin{array}{l}\text { gehlenite (syn) } \\
\left(\mathrm{Ca}_{2} \mathrm{Al}_{2} \mathrm{SiO}_{7}\right)\end{array}$ & //c-axis & ${ }^{18} \mathrm{O}$ & $1000-1300$ & 0.1 & $\mathrm{CO}_{2}$ & $186( \pm 16)$ & -11.361 & -20.4 & 498 & $\begin{array}{l}\text { Exchange with } \mathrm{C}^{18} \mathrm{O}_{2} \\
\text { atmosphere, ion probe profile }\end{array}$ & [165] \\
\hline $\begin{array}{l}\text { gehlenite (syn) } \\
\left(\mathrm{Ca}_{2} \mathrm{Al}_{2} \mathrm{SiO}_{7}\right)\end{array}$ & // a-axis & ${ }^{18} \mathrm{O}$ & $1000-1300$ & 0.1 & $\mathrm{CO}_{2}$ & $300( \pm 37)$ & -5.157 & -19.8 & 566 & $\begin{array}{l}\text { Exchange with } \mathrm{C}^{18} \mathrm{O}_{2} \\
\text { atmosphere, ion probe profile }\end{array}$ & [165] \\
\hline $\begin{array}{l}\text { melilite }(\text { syn }) \\
\left(\mathrm{Ak}_{50} \mathrm{Ch}_{50}\right)\end{array}$ & powder & ${ }^{18} \mathrm{O}$ & $799-1300$ & 0.1 & $\mathrm{CO}_{2}$ & $140.2( \pm 0.4)$ & -9.066 & -15.9 & 227 & $\begin{array}{l}\text { Exchange with } \mathrm{C}^{18} \mathrm{O}_{2} \text {, bulk } \\
\text { analysis, spherical model }\end{array}$ & {$[84]$} \\
\hline $\begin{array}{l}\text { melilite (syn) } \\
\left(\mathrm{Ak}_{75} \mathrm{Ch}_{25}\right)\end{array}$ & powder & ${ }^{18} \mathrm{O}$ & $799-1300$ & 0.1 & $\mathrm{CO}_{2}$ & $133.5( \pm 0.4)$ & -9.143 & -15.6 & 206 & $\begin{array}{l}\text { Exchange with } \mathrm{C}^{18} \mathrm{O}_{2} \text {, bulk } \\
\text { analysis, sphcrical model }\end{array}$ & {$[84]$} \\
\hline $\begin{array}{l}\text { åkermanite - } \\
\text { gehlenite } \\
\text { couple }\end{array}$ & $/ / c$-axis & $\begin{array}{l}\text { AlAl-MgSi } \\
\text { interdiffusion }\end{array}$ & $\begin{array}{l}1200 \\
1250\end{array}$ & 0.1 & $\mathrm{~N}_{2}$ & & $\begin{array}{l}D=3.9 \times 10^{-19} \\
D=6.9 \times 10^{-18}\end{array}$ & & & $\begin{array}{l}\text { EDXA profile of cross } \\
\text { section, } D=f(\text { composition }), \\
\text { maximum D reported here }\end{array}$ & [127] \\
\hline epidote & powder & ${ }^{2} \mathrm{H}$ & $450-650$ & $\begin{array}{l}200 \& \\
400\end{array}$ & UB & 57.7 & -9.48 & -12.3 & -56 & $\begin{array}{l}\text { Enchange with }\left({ }^{2} \mathrm{H},{ }^{1} \mathrm{H}\right)_{2} \mathrm{O}, \\
\text { bulk analysis, "cylinder" }\end{array}$ & {$[70]$} \\
\hline zoisite & powder & ${ }^{2} \mathrm{H}$ & $350-650$ & $\begin{array}{l}200 \& \& \\
400\end{array}$ & UB & 102.5 & -8.35 & -13.3 & 79 & $\begin{array}{l}\text { Enchange with }\left({ }^{2} \mathrm{H},{ }^{1} \mathrm{H}\right)_{2} \mathrm{O}, \\
\text { bulk analysis, "cylinder" }\end{array}$ & {$[70]$} \\
\hline $\begin{array}{l}\text { olivine } \\
\left(\mathrm{Fo}_{91} \mathrm{Fa}_{9}\right)\end{array}$ & //a-axis & "H" & $800-1000$ & $\begin{array}{l}300 \\
\text { "wet" }\end{array}$ & IW & $130( \pm 30)$ & $-4.222( \pm 0.18)$ & -10.5 & 80 & $\begin{array}{l}\text { Exchange with water, IR step } \\
\text { profiles of cross section slices }\end{array}$ & [118] \\
\hline $\begin{array}{l}\text { olivine } \\
\left(\mathrm{Fo}_{89.2}\right)\end{array}$ & powder & $\begin{array}{l}\text { Ie "apparent } \\
\text { diffusivity" }\end{array}$ & $700-1400$ & 0.0 & UB & $420( \pm 20)$ & $1.1( \pm 0.7)$ & -19.3 & 610 & $\begin{array}{l}\text { Degassing experiment, } \\
\text { spherical model, fractures! }\end{array}$ & [151] \\
\hline $\begin{array}{l}\text { olivine } \\
\left(\mathrm{Fo}_{\approx 92} \mathrm{Fa}_{-8}\right)\end{array}$ & $/ / \mathrm{c}$-axis & ${ }^{18} \mathrm{O}$ & $1200-1400$ & 0.1 & $\begin{array}{l}\text { IW \& } \\
\text { NO }\end{array}$ & $266( \pm 11)$ & $\begin{array}{l}-9.585+0.21 x \\
\log _{10}\left(f_{\mathrm{O} 2}\right) \\
\left(\mathrm{f}_{\mathrm{O} 2} \text { in } \mathrm{Pa}\right)\end{array}$ & -22.5 & 691 & $\begin{array}{l}\text { Exchange with mixed } \mathrm{C}^{18} \mathrm{O}_{2} \\
\text { gas, }{ }^{18} \mathrm{O}(p, \alpha)^{15} \mathrm{~N} \text { nuclear } \\
\text { microanalysis profiles }\end{array}$ & [142] \\
\hline $\begin{array}{l}\text { olivine } \\
\left(\mathrm{Fo}_{z 90} \mathrm{Fa}_{=10}\right)\end{array}$ & $/ / c$-axis & ${ }^{18} \mathrm{O}$ & $1090-1500$ & 0.1 & $\begin{array}{l}10^{-12-} \\
10^{-8}\end{array}$ & $318(+17)$ & $\begin{array}{l}-5.174+0.34 \mathrm{x} \\
\left.\log _{10}(\mathrm{P} 2)\right) \\
\left(\mathrm{P}_{\mathrm{O} 2} \text { in } \mathrm{Pa}\right) \\
\end{array}$ & -20.7 & 616 & $\begin{array}{l}\text { Exchange with mixed } \mathrm{H}_{2}{ }^{18} \mathrm{O} \\
\text { gas, }{ }^{18} \mathrm{O}(p, \alpha)^{15} \mathrm{~N} \text { nuclear } \\
\text { microanalysis profiles }\end{array}$ & $\lceil 61\rceil$ \\
\hline $\begin{array}{l}\text { olivine } \\
\left(\mathrm{Fo}_{90} \mathrm{Fa}_{10}\right)\end{array}$ & $\begin{array}{l}/ / \text { a-axis \& } \\
/ / \text { c-axis }\end{array}$ & ${ }^{30} \mathrm{Si}$ & $1130-1530$ & 0.1 & $\begin{array}{l}10^{-5} \\
10^{-15} \\
\end{array}$ & $291( \pm 15)$ & \begin{tabular}{|l}
$-12.735( \pm 0.18)-$ \\
$.19 x \ln \left(\mathrm{P}_{\mathrm{O} 2} / \mathrm{P}_{\mathrm{O}}\right)$ \\
\end{tabular} & -26.9 & 1047 & $\begin{array}{l}\text { Thin Fo film, Ruther-ford } \\
\text { back-scattering profiles }\end{array}$ & [90] \\
\hline $\begin{array}{l}\text { olivine } \\
\left(\mathrm{Fo}_{90} \mathrm{Fa}_{10}\right)\end{array}$ & $/ / \mathrm{c}$-axis & $\begin{array}{l}\mathrm{Ca} \\
\text { interdiffusion }\end{array}$ & $1220-1350$ & 0.1 & $10^{-9}$ & 176 & -9.155 & -17.7 & 353 & $\begin{array}{l}\text { Cation exchange with basalt, } \\
\text { microprobe profiles }\end{array}$ & [100] \\
\hline $\begin{array}{l}\text { olivinc } \\
\left(\mathrm{Fo}_{90} \mathrm{Fa}_{10}\right)\end{array}$ & $/ / \mathrm{c}$-axis & $\begin{array}{l}\mathrm{Mn} \\
\text { interdiffusion }\end{array}$ & $1220-1350$ & 0.1 & $10^{-9}$ & 218 & -7.167 & -17.8 & 410 & $\begin{array}{l}\text { Cation exchange with basalt, } \\
\text { microprobe profiles }\end{array}$ & [100] \\
\hline $\begin{array}{l}\text { olivine } \\
\left(\mathrm{Fo}_{90} \mathrm{Fa}_{10}\right)\end{array}$ & $/ / c$-axis & $\begin{array}{l}\text { Fe } \\
\text { interdiffusion }\end{array}$ & $1220-1350$ & 0.1 & $10^{-9}$ & 247 & -8.000 & -20.0 & 539 & $\begin{array}{l}\text { Cation exchange with basalt, } \\
\text { microprobe profiles }\end{array}$ & [100] \\
\hline
\end{tabular}




\begin{tabular}{|c|c|c|c|c|c|c|c|c|c|c|c|}
\hline $\begin{array}{l}\text { olivine } \\
\left(\mathrm{Fo}_{90} \mathrm{Fa}_{10}-\mathrm{Fa}_{100)}\right)\end{array}$ & $\begin{array}{l}\text { sintered } \\
\text { powder }\end{array}$ & ${ }^{59} \mathrm{Fe}$ & 1130 & 0.1 & $\begin{array}{l}10^{-10} \\
10^{-12} \\
\end{array}$ & n.d. & n.d. & & & $\begin{array}{l}\log D_{59 \mathrm{Fe}}=-10.143+0.2 \\
\mathrm{a}_{02}+2.705[\mathrm{Fe} /(\mathrm{Fe}+\mathrm{Mg})]\end{array}$ & [85] \\
\hline $\begin{array}{l}\text { olivine } \\
\left(\mathrm{Fo}_{93.7} \mathrm{Fa}_{6.3}\right)\end{array}$ & $\not / c$-axis & $\mathrm{Ni}$ & $1149-1234$ & $\begin{array}{l}\text { evac. } \\
\text { tube }\end{array}$ & UB & $193( \pm 10)$ & $-8.959( \pm 0.36)$ & -18.4 & 404 & $\begin{array}{l}\text { Thin Ni film, microprobe } \\
\text { profile, anisotropy found }\end{array}$ & [26] \\
\hline $\begin{array}{l}\text { olivine }\left(\mathrm{Fo}_{92} \mathrm{Fa}_{8}\right) \\
\text { fayalite powder }\end{array}$ & $\begin{array}{l}/ / \text { c-axis } \\
\text { (couple) }\end{array}$ & $\begin{array}{l}\text { Fe-Mg } \\
\text { interdiffusion }\end{array}$ & $1125-1200$ & 0.1 & $10^{-13}$ & 243 & -5.759 & -17.6 & 432 & $\begin{array}{l}\text { Microprobe profile, } D \text { varies } \\
\text { w/direction, composition, } f_{\mathrm{O} 2}\end{array}$ & [14] \\
\hline $\begin{array}{l}\text { olivine }\left(\mathrm{Fa}_{97} \mathrm{Te}_{3}\right) \\
\text { olivine }\left(\mathrm{Fo}_{91} \mathrm{Fa}_{9}\right)\end{array}$ & $\begin{array}{l}/ / \text { c-axis } \\
\text { (couple) }\end{array}$ & $\begin{array}{l}\text { Fe-Mg } \\
\text { interdiffusion }\end{array}$ & $900-1100$ & $\begin{array}{l}\text { evac. } \\
\text { tube }\end{array}$ & UB & $\begin{array}{l}208.5( \pm 18.8) \\
+9.1 \times[\mathrm{Mg} / \\
(\mathrm{Mg}+\mathrm{Fe})]\end{array}$ & $\begin{array}{l}\mathrm{D}_{0}=1.5( \pm 0.3) \\
\times 10^{-4)}-1.1 \mathrm{x} \\
{[\mathrm{Mg} /(\mathrm{Mg}+\mathrm{Fe})]}\end{array}$ & & & $\begin{array}{l}\text { Microprobe profile, } D \text { also } \\
\text { varies with direction and } P \\
\left(\Delta V_{a}=5.5 \times 10^{-6} \mathrm{~m}^{3} / \mathrm{mole}\right)\end{array}$ & [124] \\
\hline $\begin{array}{l}\text { olivine }\left(\mathrm{Mg}_{2} \mathrm{SiO}_{4}\right) \\
-\left(\mathrm{CO}_{2} \mathrm{SiO}_{4}\right) \\
\end{array}$ & $\begin{array}{l}/ / c \text {-axis } \\
\text { (couple) } \\
\end{array}$ & $\begin{array}{l}\text { Co-Mg } \\
\text { interdiffusion }\end{array}$ & $\begin{array}{l}1150-1300 \\
1300-1400 \\
\end{array}$ & 0.1 & air & $\begin{array}{l}196 \\
526\end{array}$ & $\begin{array}{l}-8.690 \\
2.288 \\
\end{array}$ & $\begin{array}{l}-18.2 \\
-23.3 \\
\end{array}$ & $\begin{array}{l}403 \\
781 \\
\end{array}$ & $\begin{array}{l}\text { Microprobe profile, D's } \\
\text { extrapolated to pure Fo }\end{array}$ & [125] \\
\hline \begin{tabular}{l|} 
forsterite - \\
liebensbergite
\end{tabular} & $\begin{array}{l}/ / \text { c-axis } \\
\text { (couple) }\end{array}$ & $\begin{array}{l}\mathrm{Ni}-\mathrm{Mg} \\
\text { interdiffusion }\end{array}$ & $1200-1450$ & 0.1 & air & 414 to 444 & -1.652 & -21.8 & 702 & $\begin{array}{l}\text { Microprobe profile, other } \\
\text { interdiffusion coefficients }\end{array}$ & $\begin{array}{l}{[126]} \\
{[128]}\end{array}$ \\
\hline $\begin{array}{l}\text { grossularite }\left(\mathrm{Ca}_{2.9}\right. \\
\left.\mathrm{Fe}_{.1} \mathrm{Al}_{2.0} \mathrm{Si}_{3.0} \mathrm{O}_{12}\right)\end{array}$ & isotropic & ${ }^{18} \mathrm{O}$ & $\begin{array}{l}1050 \\
850 \\
\end{array}$ & $\begin{array}{l}800 \\
200 \\
\end{array}$ & UB & $\begin{array}{l}\text { n.d. } \\
\text { n.d. }\end{array}$ & $\begin{array}{l}D=2.5 \times 10^{-20} \\
D=4.8 \times 10^{-21}\end{array}$ & & & $\begin{array}{l}\text { Exchange with }{ }^{18} \mathrm{O} \text {-cnriched } \\
\text { water, ion probe profiles }\end{array}$ & [58] \\
\hline $\begin{array}{l}\text { almandine } \\
\left(\mathrm{Al}_{67} \mathrm{Sp}_{28} \mathrm{An}_{3} \mathrm{Py}_{2}\right)\end{array}$ & isotropic & ${ }^{18} \mathrm{U}$ & $800-1000$ & 100 & UB & $301( \pm 46)$ & $-8.222( \pm 0.740)$ & -22.9 & 125 & $\begin{array}{l}\text { Exchange with }{ }^{18} \mathrm{O} \text {-enriched } \\
\text { water, ion probe profiles }\end{array}$ & [31] \\
\hline $\begin{array}{l}\text { pyrope } \\
\left(\mathrm{Py}_{74} \mathrm{Al}_{15} \mathrm{Gr}_{10} \mathrm{Ur}_{1}\right)\end{array}$ & isotropic & ${ }^{25} \mathrm{Mg}$ & $750-900$ & 200 & $\begin{array}{l}\text { UB/ } \\
\mathrm{MH}\end{array}$ & $239( \pm 16)$ & -8.009 & -19.6 & 514 & $\begin{array}{l}\text { Thin }{ }^{25} \mathrm{MgO} \text { film, ion probe } \\
\text { profiles }\end{array}$ & [34] \\
\hline $\begin{array}{l}\text { Alm }_{80} \text { Pyp }_{20}- \\
\text { Spess94 }_{94} \text { Ilm }_{6}\end{array}$ & $\begin{array}{l}\text { isotropic } \\
\text { (couple) }\end{array}$ & $\mathrm{Fe}$ & $1300-1480$ & $\begin{array}{l}2900 \\
4300\end{array}$ & $\begin{array}{l}\mathrm{UB} / \\
\mathrm{GrPC}\end{array}$ & $\begin{array}{l}275.43 \\
( \pm 36.49)\end{array}$ & -7.194 & -20.6 & 588 & $\begin{array}{l}\text { Calculated from interdiffusion } \\
\text { cxpcriments using model, } \\
\left(\Delta \mathrm{V}_{\mathrm{a}}=5.6 \times 10^{-6} \mathrm{~m}^{3} / \mathrm{mole}\right)\end{array}$ & [20] \\
\hline $\begin{array}{l}\text { Alm }_{80} \text { Pyp }_{20}- \\
\text { Spess }_{94} \text { Alm }_{6}\end{array}$ & $\begin{array}{l}\text { isotropic } \\
\text { (couple) }\end{array}$ & $\mathrm{Mg}$ & $1300-1480$ & $\begin{array}{l}2900 \\
4300\end{array}$ & $\begin{array}{l}\mathrm{UB} / \\
\mathrm{GrPC}\end{array}$ & $\begin{array}{l}284.52 \\
( \pm 37.55)\end{array}$ & -6.959 & -20.8 & 604 & $\begin{array}{l}\text { Calculated from interdiffusion } \\
\text { experiments, }\left(\Delta \mathrm{V}_{\mathrm{a}}=\right. \\
\left.5.6( \pm 2.9) \times 10^{-6} \mathrm{~m}^{3} / \text { mole }\right)\end{array}$ & [20] \\
\hline $\begin{array}{l}\text { Alm }_{80} \text { Pyp }_{20}- \\
\text { Spess }_{94} \text { Alm }_{6}\end{array}$ & $\begin{array}{l}\text { isotropic } \\
\text { (couple) }\end{array}$ & $\mathrm{Mn}$ & $1300-1480$ & $\begin{array}{l}2900- \\
4300\end{array}$ & $\begin{array}{l}\text { [IB/ } \\
\text { GrPC }\end{array}$ & $\begin{array}{l}253.44 \\
( \pm 37.19)\end{array}$ & -7.292 & -19.6 & 526 & $\begin{array}{l}\text { Calculated from interdiffusion } \\
\text { experiments, }\left(\Delta V_{a}=\right. \\
\left.5.3( \pm 3.0) \times 10^{-6} \mathrm{~m}^{3} / \text { mole }\right)\end{array}$ & {$[20]$} \\
\hline $\begin{array}{l}\text { almandine } \\
\left(\mathrm{Al}_{67} \mathrm{Sp}_{28} \mathrm{An}_{3} \mathrm{Py}_{2}\right)\end{array}$ & isotropic & ${ }^{86} \mathrm{Sr}$ & $800-1000$ & 100 & UB & $205( \pm 17)$ & $-12.000( \pm 0.602)$ & -22.0 & 616 & $\begin{array}{l}\text { Exchange with }{ }^{86} \mathrm{Sr} \text { water } \\
\text { solution, ion probe profiles }\end{array}$ & [31] \\
\hline $\begin{array}{l}\text { almandine } \\
\left(\mathrm{Al}_{67} \mathrm{Sp}_{28} \mathrm{An}_{3} \mathrm{Py}_{2}\right)\end{array}$ & isotropic & ${ }^{145} \mathrm{Nd}$ & $800-1000$ & 100 & UB & $184( \pm 29)$ & $-12.523( \pm 0.602)$ & -21.5 & 562 & $\begin{array}{l}\text { Exchange with }{ }^{145} \mathrm{Nd} \text { water } \\
\text { solution, ion probe profiles }\end{array}$ & [31] \\
\hline pyrope & powder & ${ }^{151} \mathrm{Sm}$ & $1300-1500$ & 3000 & $\begin{array}{l}\mathrm{UB} / \\
\mathrm{GrPC}\end{array}$ & 140 & -11.585 & -18.4 & 321 & $\begin{array}{l}\text { Exchange with silicate melt, } \\
\text { autoradiography, "sphere" }\end{array}$ & {$[80]$} \\
\hline $\begin{array}{l}\text { almandine } \\
\left(\mathrm{Al}_{67} \mathrm{Sp}_{28} \mathrm{An}_{3} \mathrm{Py}_{2}\right)\end{array}$ & isulropic & ${ }^{167} \mathrm{EI}_{\mathrm{I}}$ & $800-1000$ & 100 & $\mathrm{UB}$ & $230( \pm 38)$ & $-10.301( \pm 0.763)$ & -21.5 & 605 & $\begin{array}{l}\text { Exchange with }{ }^{167} \mathrm{Er} \text { water } \\
\text { solution, ion probe profiles }\end{array}$ & [31] \\
\hline $\begin{array}{l}\mathrm{Alm}_{80} \mathrm{Pyp}_{20}- \\
\text { Spess }_{94} \mathrm{Alm}_{6}\end{array}$ & $\begin{array}{l}\text { isotropic } \\
\text { (couple) }\end{array}$ & $\begin{array}{l}\text { Fe-Mn } \\
\text { interdiffusion }\end{array}$ & $1300-1480$ & 4000 & $\begin{array}{l}\mathrm{UB} / \\
\mathrm{GrPC}\end{array}$ & $\begin{array}{l}224.3 \\
( \pm 20.5)\end{array}$ & -10.086 & -21.0 & 571 & $\begin{array}{l}\text { Microprobe profiles, model } \\
\text { fits of alm-rich composition }\end{array}$ & [41] \\
\hline
\end{tabular}




\begin{tabular}{|c|c|c|c|c|c|c|c|c|c|c|c|}
\hline $\begin{array}{c}\text { Mineral, Glass, } \\
\text { or Liquid }\end{array}$ & $\begin{array}{l}\text { Orienta- } \\
\text { tion }\end{array}$ & $\begin{array}{c}\text { Diffusing } \\
\text { Component }\end{array}$ & $\begin{array}{l}\text { Temperature } \\
\text { Range }\left({ }^{\circ} \mathrm{C}\right)\end{array}$ & $\begin{array}{c}\mathrm{P} \\
(\mathrm{MPa})\end{array}$ & $\begin{array}{c}\mathrm{O}_{2} \\
(\mathrm{MPa})\end{array}$ & $\begin{array}{c}\Delta \mathrm{E}_{\mathrm{a}} \\
(\mathrm{kJ} / \mathrm{mole})\end{array}$ & $\begin{array}{c}\log D_{o}(\text { or } D) \\
\left(\mathrm{m}^{2} / \mathrm{s}\right)\end{array}$ & \begin{tabular}{|c|}
$\log \mathrm{D}$ \\
$1200^{\circ} \mathrm{C}$ \\
\end{tabular} & $\begin{array}{l}\text { "Tc" } \\
\left({ }^{\circ} \mathrm{C}\right) \\
\end{array}$ & Experiment/Comments & Ref. \\
\hline
\end{tabular}

\begin{tabular}{|c|c|c|c|c|c|c|c|c|c|c|c|}
\hline titanite & $/ / c$-axis & ${ }^{18} \mathrm{O}$ & $700-900$ & 100 & $\begin{array}{l}\text { UB/ } \\
\text { NO }\end{array}$ & 301 & -5.638 & -20.3 & 591 & $\begin{array}{l}\text { Exchange with }{ }^{18} \mathrm{O} \text {-enriched } \\
\text { water, ion probe profiles }\end{array}$ & [129] \\
\hline titanite & $/ / \mathrm{c}$-axis & ${ }^{86} \mathrm{Sr}$ & $700-900$ & 100 & $\begin{array}{l}\mathrm{UB} / \\
\mathrm{NO} \\
\end{array}$ & 234 & -9.420 & -20.8 & 570 & $\begin{array}{l}\text { Exchange with }{ }^{18} \mathrm{O} \text {-enriched } \\
\text { water, ion probe profiles }\end{array}$ & {$[129]$} \\
\hline titanite & $/ /(100)$ & $\mathrm{Pb}$ & $650-1027$ & 0.1 & air & $328.5( \pm 11.3)$ & $-3.955( \pm 0.315)$ & -19.9 & 591 & $\begin{array}{l}\text { Exchange with PbS powder, } \\
\text { Rutherford backscattering }\end{array}$ & [22] \\
\hline zircon & n.d. & $\mathrm{Pb}$ & $550-800$ & 0.1 & air & $142( \pm 8)$ & -11.699 & -18.6 & 337 & $\begin{array}{l}\text { Ion implantation of } \mathrm{Pb}, \mathrm{Ru}- \\
\text { therford backscattering }\end{array}$ & [23] \\
\hline rhyolite & glass & $\mathrm{Li}$ & $297-909$ & 0.1 & air & $92.1( \pm 1.3)$ & $-5.599( \pm 0.079)$ & -8.9 & & $\begin{array}{l}\text { Thin film of } \mathrm{LiNO}_{3} \text {, ion probe } \\
\text { profile on cross section }\end{array}$ & {$[92]$} \\
\hline $\begin{array}{l}\text { rhyolite (obsidian) } \\
\text { Iceland }\end{array}$ & glass & ${ }^{24} \mathrm{Na}$ & $140-850$ & 0.1 & air & $84.5( \pm 1.3)$ & $-5.91( \pm 0.18)$ & -8.9 & & $\begin{array}{l}\text { Thin film, serial sectioning by } \\
\text { etching, counting surface }\end{array}$ & [91] \\
\hline "haplogranite" & melt & $\begin{array}{l}\text { "B-Si" } \\
\text { interdiffusion }\end{array}$ & $1200-1600$ & 0.1 & n.d. & $288.5( \pm 20.4)$ & $-4.864( \pm 0.640)$ & -15.1 & & $\begin{array}{l}\text { Ion probe profile of cross } \\
\text { section }\end{array}$ & {$[18]$} \\
\hline $\begin{array}{l}\text { rhyolite(obsidian) } \\
\text { Lake County, OR }\end{array}$ & melt & $\mathrm{P}$ & $1200-1500$ & 800 & $\begin{array}{l}\mathrm{UB} / \\
\mathrm{GrPC}\end{array}$ & $600.9( \pm 11.7)$ & $-12.652( \pm 0.334)$ & -34.0 & & $\begin{array}{l}\text { Apatite dissolution, } \\
\text { microprobe profile, effect of } \\
\text { water measured }\end{array}$ & {$[79]$} \\
\hline $\begin{array}{l}\text { rhyolite (obsidian) } \\
\text { Lake County, OR }\end{array}$ & \begin{tabular}{|l|} 
melt with \\
$8 \%$ water \\
\end{tabular} & ${ }^{36} \mathrm{Cl}$ & 1100 & 1000 & $\begin{array}{l}\mathrm{UB} / \\
\mathrm{GrPC}\end{array}$ & & $\mathrm{D}=1.29 \times 10^{-11}$ & & & $\begin{array}{l}\text { Thin film of } \mathrm{Na}^{36} \mathrm{Cl}, \beta \text {-track } \\
\text { profiles of cross section }\end{array}$ & [155] \\
\hline $\begin{array}{l}\text { rhyolite (obsidian) } \\
\text { Iceland }\end{array}$ & glass & ${ }^{42} \mathrm{~K}$ & $350-850$ & 0.1 & air & $106.3( \pm 3.8)$ & $-6.46( \pm 0.24)$ & -10.2 & & $\begin{array}{l}\text { Thin film, serial sectioning by } \\
\text { etching, counting surface }\end{array}$ & [91] \\
\hline $\begin{array}{l}\text { rhyolite (obsidian) } \\
\text { Iceland }\end{array}$ & glass & $\mathrm{Ca}$ & $630-930$ & 0.1 & air & $283.7( \pm 4.6)$ & $-0.69( \pm 0.22)$ & -10.7 & & $\begin{array}{l}\text { Thin film, serial sectioning by } \\
\text { etching, counting surface }\end{array}$ & {$[91]$} \\
\hline $\begin{array}{l}\text { rhyolite (obsidian) } \\
\text { Iceland }\end{array}$ & glass & ${ }^{86} \mathrm{Rb}$ & $400-950$ & 0.1 & air & $127.2( \pm 0.8)$ & $-6.86( \pm 0.05)$ & -11.4 & & $\begin{array}{l}\text { Thin film, serial sectioning by } \\
\text { etching, counting surface }\end{array}$ & [91] \\
\hline \begin{tabular}{l|} 
rhyolite \\
(dehydrated) NM
\end{tabular} & glass & ${ }^{85} \mathrm{Sr}$ & $650-950$ & 0.1 & air & $178.7( \pm 3.3)$ & $-5.260(+0.175)$ & -11.6 & & $\begin{array}{l}\text { Thin film, serial sectioning by } \\
\text { grinding, counting surface }\end{array}$ & {$[119]$} \\
\hline $\begin{array}{l}\text { thyolite (obsidian) } \\
\text { Lake County, OR }\end{array}$ & melt(dry) & $\mathrm{Zr}$ & $1020-1500$ & 800 & $\begin{array}{l}\mathrm{UB} / \\
\mathrm{GrPC}\end{array}$ & $408.8( \pm 11.7)$ & $-1.009( \pm 0.386$ & -15.5 & & $\begin{array}{l}\text { Zircon dissolution, } \\
\text { microprobe profiles }\end{array}$ & [78] \\
\hline $\begin{array}{l}\text { rhyolite (obsidian) } \\
\text { Lake County, OR }\end{array}$ & melt(wet) & $\mathrm{Zr}$ & $1020-1385$ & 800 & $\begin{array}{l}\mathrm{UB} / \\
\mathrm{GrPC}\end{array}$ & $197.9( \pm 8.0)$ & $-5.523( \pm 0.301)$ & -12.5 & & $\begin{array}{l}\text { Zircon dissolution, } \\
\text { microprobe profiles }\end{array}$ & {$[78]$} \\
\hline $\begin{array}{l}\text { rhyolite (obsidian) } \\
\text { Iccland }\end{array}$ & glass & ${ }^{134} \mathrm{Cs}$ & $600-920$ & 0.1 & air & $208.4( \pm 8.4)$ & $-5.04( \pm 0.44)$ & -12.4 & & $\begin{array}{l}\text { Thin film, serial sectioning by } \\
\text { etching, counting surface }\end{array}$ & [91] \\
\hline \begin{tabular}{l|} 
rhyolite \\
(dehydrated) NM
\end{tabular} & $\begin{array}{l}\text { glass and } \\
\text { melt }\end{array}$ & ${ }^{134} \mathrm{Cs}$ & $790-1300$ & 0.1 & air & $\begin{array}{l}201.3 \\
( \pm 12.1) \\
\end{array}$ & $-6.01( \pm 0.45)$ & -13.1 & & $\begin{array}{l}\text { Thin film, serial sectioning by } \\
\text { etching, counting surfacc }\end{array}$ & [91] \\
\hline $\begin{array}{l}\text { rhyolite (obsidian) } \\
\text { Lake County, OR }\end{array}$ & melt & ${ }^{134} \mathrm{Cs}$ & $700-800$ & 210 & $\begin{array}{l}\mathrm{UB} / \\
\mathrm{NO} \\
\end{array}$ & 81.68 & -8.143 & -11.0 & & $\begin{array}{l}\text { Thin film, } \beta \text {-track profiles of } \\
\text { cross section }\end{array}$ & [153] \\
\hline $\begin{array}{l}\text { rhyolite } \\
\text { (dehydrated) NM }\end{array}$ & glass & ${ }^{133} \mathrm{Ba}$ & $650-950$ & 0.1 & air & $188.7( \pm 6.3)$ & $-5.42( \pm 0.30)$ & -12.1 & & $\begin{array}{l}\text { Thin film, serial sectioning by } \\
\text { grinding, counting surface }\end{array}$ & [119] \\
\hline
\end{tabular}




\begin{tabular}{|c|c|c|c|c|c|c|c|c|c|c|c|}
\hline $\begin{array}{c}\text { Mineral, Glass, } \\
\text { or Liquid }\end{array}$ & $\begin{array}{c}\text { Orienta- } \\
\text { tion }\end{array}$ & $\begin{array}{l}\text { Diffusing } \\
\text { Componcnt } \\
\end{array}$ & $\begin{array}{r}\text { Temperature } \\
\text { Rangc }\left({ }^{\circ} \mathrm{C}\right)\end{array}$ & $\begin{array}{c}\mathrm{P} \\
(\mathrm{MPa})\end{array}$ & $\begin{array}{c}\mathrm{O}_{2} \\
(\mathrm{MPa})\end{array}$ & $\begin{array}{c}\Delta \mathrm{E}_{\mathrm{a}} \\
(\mathrm{kJ} / \mathrm{mole})\end{array}$ & $\begin{array}{c}\log D_{0}(\text { or } D) \\
\left(\mathrm{m}^{2} / \mathrm{s}\right) \\
\end{array}$ & $\begin{array}{r}\log \mathrm{D} \\
1200^{\circ} \mathrm{C} \\
\end{array}$ & $\begin{array}{l}" \mathrm{~T}_{\mathrm{c}} " \\
\left({ }^{\circ} \mathrm{C}\right) \\
\end{array}$ & Experiment/Comments & Ref. \\
\hline $\begin{array}{l}\text { rhyolite } \\
\text { (dehydrated) NM }\end{array}$ & $\begin{array}{l}\text { glass and } \\
\text { melt }\end{array}$ & $\mathrm{Ce}$ & $875-1100$ & 0.1 & air & $\begin{array}{l}490.4 \\
( \pm 23.9)\end{array}$ & $2.72( \pm 0.99)$ & -14.7 & & $\begin{array}{l}\text { Thin film, serial sectioning by } \\
\text { etching, counting surface }\end{array}$ & [91] \\
\hline $\begin{array}{l}\text { rhyolite } \\
\text { (dehydrated) NM }\end{array}$ & $\begin{array}{l}\text { glass and } \\
\text { melt }\end{array}$ & Eu & $700-1050$ & $\overline{0.1}$ & air & $288.7( \pm 5.0)$ & $-3.11(0.22)$ & -13.3 & & $\begin{array}{l}\text { Thin film, serial sectioning by } \\
\text { etching, counting surface }\end{array}$ & [91] \\
\hline $\begin{array}{l}\text { rhyolite (obsidian) } \\
\text { Lake County, OR }\end{array}$ & \begin{tabular}{|l|l|}
$\begin{array}{l}\text { melt } 6 \% \\
\text { water }\end{array}$ \\
\end{tabular} & LREE & $1000-1400$ & 800 & $\begin{array}{l}\mathrm{UB} / \\
\mathrm{GrPC}\end{array}$ & $251.5( \pm 42.3)$ & $-4.638( \pm 1.436)$ & -13.6 & & $\begin{array}{l}\text { Monazite dissolution, } \\
\text { microprobe profiles }\end{array}$ & [138] \\
\hline $\begin{array}{l}\text { rhyolite (obsidian) } \\
\text { Lake County, OR }\end{array}$ & $\begin{array}{l}\text { melt } 1 \% \\
\text { water }\end{array}$ & LREE & $1000-1400$ & 800 & $\begin{array}{l}\mathrm{UB} / \\
\mathrm{GrPC}\end{array}$ & $510.9( \pm 59.0)$ & $3.362( \pm 0.629)$ & -14.8 & & $\begin{array}{l}\text { Monazite dissolution, } \\
\text { microprobe profiles }\end{array}$ & [138] \\
\hline $\begin{array}{l}\text { rhyolite (obsidian) } \\
\text { Mono Craters }\end{array}$ & glass & $\mathrm{H}_{2} \mathrm{O}$ & $400-850$ & 0.1 & $\mathrm{~N}_{2}$ & $103( \pm 5)$ & $-14.59( \pm 1.59)$ & -18.2 & & $\begin{array}{l}\text { Dehydration in } \mathrm{N}_{2} \text {, FTIR } \\
\text { profile, equilibrium model }\end{array}$ & [166] \\
\hline $\begin{array}{l}\text { rhyolite (obsidian) } \\
\text { New Mexico }\end{array}$ & glass & $\mathrm{H}_{2} \mathrm{O}$ & $510-980$ & 0.1 & air & $\begin{array}{l}46.48 \\
( \pm 11.40)\end{array}$ & $-10.90( \pm 0.56)$ & -12.5 & & $\begin{array}{l}\text { Dehydration in air, bulk } \\
\text { weight loss, low water }\end{array}$ & [93] \\
\hline $\begin{array}{l}\text { rhyolite (obsidian) } \\
\text { Lake County, OR }\end{array}$ & $\begin{array}{l}\text { melt with } \\
8 \% \text { water }\end{array}$ & ${ }^{14} \mathrm{CO}_{2}$ & $800-1100$ & 1000 & $\begin{array}{l}\text { UB/ } \\
\text { GrPC }\end{array}$ & $75( \pm 21)$ & -7.187 & -9.9 & & $\begin{array}{l}\text { Thin film of } \mathrm{Na}_{2}{ }^{14} \mathrm{CO}_{3}, \beta- \\
\text { track profiles of cross section }\end{array}$ & [155] \\
\hline & & & & & & & & & & & \\
\hline $\begin{array}{l}\text { basalt } \\
\text { (alkali) }\end{array}$ & melt & ${ }^{6} \mathrm{Li}$ & $1300-1400$ & 0.1 & air & 115.5 & -5.125 & -9.2 & & $\begin{array}{l}\text { Thin film of }{ }^{6} \mathrm{I}, \mathrm{iCl} \text {, ion probe } \\
\text { profile of cross section }\end{array}$ & [116] \\
\hline $\begin{array}{l}\text { basalt } \\
\text { Goose Island }\end{array}$ & melt & $\mathrm{O}$ & $1160-1360$ & 0.1 & $\begin{array}{l}\text { IW to } \\
\mathrm{CO}_{2}\end{array}$ & $\begin{array}{l}215.9 \\
( \pm 13.4) \\
\end{array}$ & -2.439 & -10.1 & & $\begin{array}{l}\text { Oxidation/reduction of bead, } \\
\text { thermo-gravimetric balance }\end{array}$ & {$[160]$} \\
\hline $\begin{array}{l}\text { basalt (alkali } \\
\text { olivine) } \mathrm{BC}\end{array}$ & & & $\begin{array}{l}1280-1400 \\
-\quad-\quad-\end{array}$ & 400 & $\begin{array}{l}\text { UB/ } \\
\text { GrPC }\end{array}$ & $293( \pm 29)$ & $-0.790( \pm 2.51)$ & -11.2 & & $\begin{array}{l}\text { Reduction by graphite, bulk } \\
\mathrm{FeO} \text { analysis by titration }\end{array}$ & {$[40]$} \\
\hline $\begin{array}{l}\text { basalt (alkali } \\
\text { olivine) } B C\end{array}$ & melt & O & $1280-1450$ & 1200 & $\begin{array}{l}\mathrm{UB} / \\
\mathrm{GrPC}\end{array}$ & $360( \pm 25)$ & $1.450( \pm 0.081)$ & -11.3 & & $\begin{array}{l}\text { Reduction by graphite, bulk } \\
\text { FeO analysis by titration }\end{array}$ & [40] \\
\hline $\begin{array}{l}\text { basalt (alkali } \\
\text { olivine) BC }\end{array}$ & melt & $\mathrm{O}$ & $1350-1450$ & 2000 & $\begin{array}{l}\text { UB/ } \\
\text { GrPC }\end{array}$ & $297( \pm 59)$ & $-0.770( \pm 1.87)$ & -11.3 & & $\begin{array}{l}\text { Reduction by graphite, bulk } \\
\text { FeO analysis by titration }\end{array}$ & {$[40]$} \\
\hline $\begin{array}{l}\text { basalt (tholeiite) } \\
1921 \text { Kilauea }\end{array}$ & melt & $\mathrm{O}$ & $1300-1450$ & 1200 & $\begin{array}{l}\mathrm{UB} / \\
\mathrm{GrPC}\end{array}$ & $213( \pm 17)$ & $-3.010( \pm 0.59)$ & -10.6 & & $\begin{array}{l}\text { Reduction by graphite, bulk } \\
\mathrm{FeO} \text { analysis by titration }\end{array}$ & [40] \\
\hline $\begin{array}{l}\text { basalt (FeTi) } \\
\text { Galapagos }\end{array}$ & melt & ${ }^{18} \mathrm{O}$ & $1320-1500$ & 0.1 & $\begin{array}{l}\mathrm{CO}_{2} \\
\& \mathrm{O}_{2}\end{array}$ & $251( \pm 29)$ & -2.854 & -11.8 & & $\begin{array}{l}\text { Exchange with }{ }^{18} \mathrm{O} \text {-selected } \\
\text { gas, bulk analysis of sphere }\end{array}$ & {$[15]$} \\
\hline $\begin{array}{l}\text { "basalt" (Fe-free) } \\
\text { (synthetic) }\end{array}$ & melt & $\mathrm{Ar}$ & $1300-1450$ & $\begin{array}{l}1000 \\
3000 \\
\end{array}$ & & $113.2( \pm 7.5)$ & $6.140( \pm 0.068)$ & -10.2 & & Method not described & [39] \\
\hline $\begin{array}{l}\text { basalt ("alkali") } \\
\text { Tenerife }\end{array}$ & melt & ${ }^{24} \mathrm{Na}$ & $1300-1400$ & 0.1 & air & $163( \pm 13)$ & $-4.02( \pm 0.46)$ & -9.8 & & $\begin{array}{l}\text { Thin film, serial sectioning by } \\
\text { grinding, counting surface }\end{array}$ & [116] \\
\hline $\begin{array}{l}\text { basalt (tholeiite) } \\
1921 \text { Kilauea }\end{array}$ & melt & ${ }^{45} \mathrm{Ca}$ & $1260-1440$ & 0.1 & air & 184.1 & -4.272 & -10.8 & & $\begin{array}{l}\text { Thin film, } \beta \text {-track profiles of } \\
\text { cross-section on film }\end{array}$ & [89] \\
\hline $\begin{array}{l}\text { "basalt" (Fe-free) } \\
\text { (synthetic) }\end{array}$ & melt & ${ }^{45} \mathrm{Ca}$ & $1100-1400$ & 0.1 & UB & 106.3 & -6.301 & -10.1 & & $\begin{array}{l}\text { Thin film of }{ }^{45} \mathrm{CaCl}, \beta \text {-track } \\
\text { profiles of cross section }\end{array}$ & [152] \\
\hline $\begin{array}{l}\text { "basalt" (Fe-free) } \\
\text { (synthetic) }\end{array}$ & melt & ${ }^{45} \mathrm{Ca}$ & $1100-1400$ & 1000 & UB & 141.0 & -5.284 & -10.3 & & $\begin{array}{l}\text { Thin film of }{ }^{45} \mathrm{CaCl}, \beta \text {-track } \\
\text { profiles of cross section }\end{array}$ & [152] \\
\hline $\begin{array}{l}\text { "basalt" (Fe-free) } \\
\text { (synthetic) }\end{array}$ & melt & ${ }^{45} \mathrm{Ca}$ & $1100-1400$ & 2000 & UB & 208.4 & -3.211 & -10.6 & & $\begin{array}{l}\text { Thin film of }{ }^{45} \mathrm{CaCl}, \beta \text {-track } \\
\text { profiles of cross section }\end{array}$ & [152] \\
\hline
\end{tabular}




\begin{tabular}{|c|c|c|c|c|c|c|c|c|c|c|c|}
\hline $\begin{array}{l}\text { Mineral, Glass, } \\
\text { or Liquid } \\
\end{array}$ & $\begin{array}{l}\text { Orienta- } \\
\text { tion }\end{array}$ & $\begin{array}{c}\text { Diffusing } \\
\text { Component }\end{array}$ & $\begin{array}{c}\text { Temperature } \\
\text { Range }\left({ }^{\circ} \mathrm{C}\right) \\
\end{array}$ & $\begin{array}{c}\mathrm{P} \\
(\mathrm{MPa}) \\
\end{array}$ & $\begin{array}{c}\mathrm{O}_{2} \\
(\mathrm{MPa})\end{array}$ & $\begin{array}{c}\Delta \mathrm{E}_{\mathrm{a}} \\
(\mathrm{kJ} / \mathrm{mole})\end{array}$ & $\begin{array}{c}\log \mathrm{D}_{\mathrm{o}} \text { (or D) } \\
\left(\mathrm{m}^{2} / \mathrm{s}\right)\end{array}$ & $\begin{array}{c}\log \mathrm{D} \\
1200^{\circ} \mathrm{C} \\
\end{array}$ & $\begin{array}{l}" \mathrm{~T}_{\mathrm{c}} " \\
\left({ }^{\circ} \mathrm{C}\right)\end{array}$ & Experiment/Comments & Ref. \\
\hline $\begin{array}{l}\text { basalt ("alkali") } \\
\text { Tenerife }\end{array}$ & melt & ${ }^{46} \mathrm{Sc}$ & $1300-1400$ & 0.1 & air & $197( \pm 8)$ & $-4.55( \pm 0.31)$ & -11.5 & & $\begin{array}{l}\text { Thin film, serial sectioning by } \\
\text { grinding, counting surface }\end{array}$ & [116] \\
\hline $\begin{array}{l}\text { basalt ("alkali") } \\
\text { Tenerife }\end{array}$ & melt & ${ }^{54} \mathrm{Mn}$ & $1300-1400$ & 0.1 & air & $201( \pm 25)$ & $-3.80( \pm 0.81)$ & -10.9 & & $\begin{array}{l}\text { Thin film, serial sectioning by } \\
\text { grinding, counting surface }\end{array}$ & [116] \\
\hline $\begin{array}{l}\text { basalt ("alkali") } \\
\text { Tenerife }\end{array}$ & melt & ${ }^{59} \mathrm{Fe}$ & $1300-1400$ & 0.1 & air & $264( \pm 17)$ & $-2.20( \pm 0.59)$ & -11.5 & & $\begin{array}{l}\text { Thin film, serial sectioning by } \\
\text { grinding, counting surface }\end{array}$ & {$[116]$} \\
\hline $\begin{array}{l}\text { basalt (tholeiite) } \\
1921 \text { Kilauea }\end{array}$ & melt & ${ }^{60} \mathrm{Co}$ & $1260-1440$ & 0.1 & air & 151.9 & -5.276 & -10.7 & & $\begin{array}{l}\text { Thin film, serial sectioning by } \\
\text { grinding, counting surface }\end{array}$ & [89] \\
\hline $\begin{array}{l}\text { basalt ("alkali") } \\
\text { Tenerife }\end{array}$ & melt & ${ }^{60} \mathrm{Co}$ & $1300-1400$ & 0.1 & air & $201( \pm 21)$ & $-3.83( \pm 0.61)$ & -11.0 & & $\begin{array}{l}\text { Thin film, serial sectioning by } \\
\text { grinding, counting surface }\end{array}$ & [116] \\
\hline $\begin{array}{l}\text { basalt (tholeiite) } \\
1921 \text { Kilauea }\end{array}$ & melt & ${ }^{85} \mathrm{Sr}$ & $1260-1440$ & 0.1 & air & 182.0 & -4.556 & -11.0 & & $\begin{array}{l}\text { Thin film, serial sectioning by } \\
\text { grinding, counting surface }\end{array}$ & [89] \\
\hline $\begin{array}{l}\text { basalt ("alkali") } \\
\text { Tenerife } \\
\end{array}$ & melt & ${ }^{85} \mathrm{Sr}$ & $1300-1400$ & 0.1 & air & $213( \pm 25)$ & $-3.46( \pm 0.83)$ & -11.0 & & $\begin{array}{l}\text { Thin film, serial sectioning by } \\
\text { grinding, counting surface }\end{array}$ & [116] \\
\hline $\begin{array}{l}\text { basalt (tholeiite) } \\
1921 \text { Kilauea }\end{array}$ & melt & ${ }^{133} \mathrm{Ba}$ & $1260-1440$ & 0.1 & air & 164.9 & -5.229 & -11.1 & & $\begin{array}{l}\text { Thin film, serial sectioning by } \\
\text { grinding, counting surface }\end{array}$ & [89] \\
\hline $\begin{array}{l}\text { basalt ("alkali") } \\
\text { Tenerife }\end{array}$ & melt & ${ }^{133} \mathrm{Ba}$ & $1300-1400$ & 0.1 & air & $172( \pm 17)$ & $-5.00( \pm 0.54)$ & -11.1 & & $\begin{array}{l}\text { Thin film, serial sectioning by } \\
\text { grinding, counting surface }\end{array}$ & [116] \\
\hline $\begin{array}{l}\text { basalt ("alkali") } \\
\text { Tenerife }\end{array}$ & melt & ${ }^{134} \mathrm{Cs}$ & $1300-1400$ & 0.1 & air & $272( \pm 17)$ & $-2.00( \pm 0.60)$ & -11.6 & & $\begin{array}{l}\text { Thin film, serial sectioning by } \\
\text { grinding, counting surface }\end{array}$ & {$[116]$} \\
\hline $\begin{array}{l}\text { basalt (tholeiite) } \\
1921 \text { Kilauea }\end{array}$ & melt & $\begin{array}{l}{ }^{152} \mathrm{Eu}, \\
{ }^{153} \mathrm{Gd}\end{array}$ & $\begin{array}{l}1320-1440 \\
1320-1210 \\
\end{array}$ & 0.1 & air & 169.9 & $\begin{array}{l}5.237 \\
D=1.4 \times 10^{-11}\end{array}$ & & & $\begin{array}{l}\text { Thin film, serial sectioning by } \\
\text { grinding, counting surface }\end{array}$ & [120] \\
\hline $\begin{array}{l}\text { "basalt" (Fe-free) } \\
\text { (synthetic) }\end{array}$ & melt & ${ }^{14} \mathrm{CO}_{2}$ & $1350-1500$ & 1500 & $\begin{array}{l}\mathrm{UB} / \\
\mathrm{GrPC}\end{array}$ & 195.0 & -3.449 & -10.4 & & $\begin{array}{l}\text { Thin film of } \mathrm{Na}^{14} \mathrm{CO}_{3}, \beta \text {-track } \\
\text { profiles of cross section, } \\
\text { pressure dependence }\end{array}$ & [159] \\
\hline
\end{tabular}

Key to oxygen fugacity or atmosphere abbreviations in data table

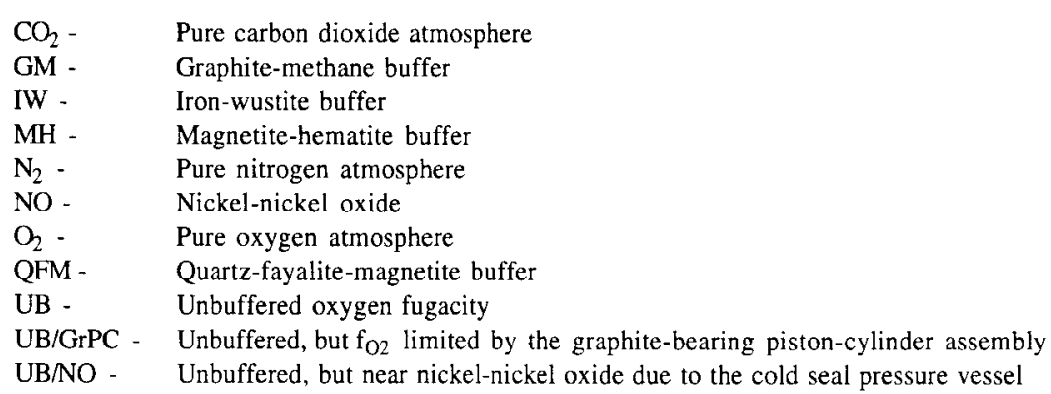




\section{REFERENCES}

1. Ashworth, J. R., Birdi, J. J., and Emmett, T. F., Diffusion in coronas around clinopyroxene: modelling with local equilibrium and steady state, and a nonsteady-state modification to account for zoned actinolitehornblende, Contrib. Mineral. Petrol., I09, 307-325, 1992.

2. Askill, J., Tracer Diffusion Data for Metals, Alloys, and Simple Oxides, 97 pp., Plenum Press, New York, 1970.

3. Baker, D. R., Interdiffusion of hydrous dacitic and rhyolitic melts and the efficacy of rhyolite contamination of dacitic enclaves, Contrib. Mineral. Petrol., 106, 462-473, 1991.

4. Baker, D. R., The effect of F and $\mathrm{Cl}$ on the interdiffusion of peralkaline intermediate and silicic melts, Am. Mineral., 78, 316-324, 1993.

5. Barrer, R. M., Bartholomew, R. F., and Rees, L. V. C., Ion exchange in porous crystals. Part II. The relationship between self- and exchange-diffusion coefficients, J. Phys. Chem. Solids, 24, 309-317, 1963.

6. Barrer, Richard M., Diffusion in and through Solids, 464 pp., Cambridge University Press, Cambridge, England, 1951.

7. Besancon, J. R., Rate of disordering in orthopyroxenes, Am. Mineral., 66, 965-973, 1981.

8. Boltzmann, L., Zur Integration der Diffusionsgleichung bei variabeln Diffusion-coefficienten Ann. Physik Chem., 53, 959964, 1894.

9. Brabander, D. J., and Giletti, B. J., Strontium diffusion kinetics in amphiboles (abstract), $A G U$ 1992 Fall Meeting, supplement to Eos Trans. $A G U, 73,641$, 1992.
10. Brady, J. B., Reference frames and diffusion coefficients, $A m$. J. Sci., 275, 954-983, 1975.

11. Brady, J. B., Intergranular diffusion in metamorphic rocks, Am. J. Sci., 283-A, 181-200, 1983.

12. Brady, J. B., and McCallister, R. H., Diffusion data for clinopyroxenes from homogenization and self-diffusion experiments, Am. Mineral., 68, 95-105, 1983.

13. Brenan, J. M., Diffusion of chlorine in fluid-bearing quartzite: Effects of fluid composition and total porosity, Contrib. Mineral. Petrol., 115, 215-224, 1993.

14. Buening, D. K., and Buseck, P. $\mathrm{R}$, $\mathrm{Fe}-\mathrm{Mg}$ lattice diffusion in olivine, J. Geophys. Res., 78, 6852-6862, 1973.

15. Canil, D., and Muehlenbachs, $\mathrm{K}$., Oxygen diffusion in an Ferich basalt melt, Geochim. Cosmochim. Acta, 54, 2947 $2951,1990$.

16. Carlson, W. D., and Johnson, C. D., Coronal reaction textures in garnet amphibolites of the Llano Uplift, Am. Mineral., 76, 756-772, 1991.

17. Carslaw, H. S., Jaeger, J. C., Conduction of Heat in Solids, 510 pp., Oxford, Clarendon Press, 1959.

18. Chakraborty, S., Dingwell, D., and Chaussidon, M., Chemical diffusivity of boron in melts of haplogranitic composition, Geochim. Cosmochim. Acta, 57, 1741-1751, 1993.

19. Chakraborty, S., and Ganguly, J., Compositional zoning and cation diffusion in garnets, in Diffusion, Atomic Ordering, and Mass Transport, edited by Ganguly, J., pp. 120-175, Springer-Verlag, New York,
1991.

20. Chakraborty, S., and Ganguly, $\mathrm{J}$, Cation diffusion in aluminosilicate garnets: experimental determination in spessartinealmandine diffusion couples, evaluation of effective binary diffusion coefficients, and applications, Contrib. Mineral. Petrol., 111, 74-86, 1992.

21. Cherniak, D. J., Diffusion of $\mathrm{Pb}$ in feldspar measured by Rutherford backscatteing spectroscopy, AGU 1992 Fall Meeting, supplement to Eos Trans. $A G U, 73,641,1992$.

22. Cherniak, D., Lead diffusion in titanite and preliminary results on the effects of radiation damage on $\mathrm{Pb}$ transport, Chem. Geol., 110, 177-194, 1993.

23. Cherniak, D. J., Lanford, W. A., and Ryerson, R. J., Lead diffusion in apatite and zircon using ion implantation and Rutherford backscattering techniques, Geochim. Cosmochim. Acta, 55, 1663-1673, 1991.

24. Cherniak, D. J., and Watson, E. B., A study of strontium diffusion in $\mathrm{K}$-feldspar, $\mathrm{Na}-\mathrm{K}$ feldspar and anorthite using Rutherford backscattering spectroscopy, Earth Planet. Sci. Lett., 113, 411-425, 1992.

25. Christoffersen, R., Yund, R. A., and Tullis, J., Interdiffusion of $\mathrm{K}$ and $\mathrm{Na}$ in alkali feldspars, Am. Mineral., 68 , 1126-1133, 1983.

26. Clark, A. M., and Long, J. V. $P$., The anisotropic diffusion of nickel in olivine, in Thomas Graham Memorial Symposium on Diffusion Processes, edited by Sherwood, J. N., Chadwick, A. V., Muir, W. M., and Swinton, F. L., pp. 511-521, Gordon and Breach, New York, 1971. 
27. Connolly, C., and Muehlenbachs, K., Contrasting oxygen diffusion in nepheline, diopside and other silicates and their relevance to isotopic systematics in meteorites, Geochim. Cosmochim. Acta, 52, 1585-1591, 1988.

28. Cooper, A. R., The use and limitations of the concept of an effective binary diffusion coefficient for multi-component diffusion, in Mass Transport in Oxides, edited by Wachtman, J. B., Jr., and Franklin, A. D., pp. 79-84, National Bureau of Standards, Washington, 1968.

29. Cooper, A. R., Jr. Vector space treatment of multicomponent diffusion, in Geochemical Transport and Kinetics, edited by Hofmann, $\Lambda$. W., Giletti, B. J., Yoder, H. S., Jr., and Yund, R. A., pp. 15-30, Carnegie Institution of Washington, Washington, 1974.

30. Cooper, A. R., and Varshneya, A. K., Diffusion in the system $\mathrm{K}_{2} \mathrm{O}-\mathrm{SrO}-\mathrm{SiO}_{2}$, effective binary diffusion coefficients, $A m$. Ceram. Soc. J., 51, 103-106, 1968.

31. Coughlan, R. A. N., Studies in diffusional transport: grain boundary transport of oxygen in feldspars, strontium and the REE's in garnet, and thermal histories of granitic intrusions in south-central Maine using oxygen isotopes, Ph.D. thesis, Brown University, Providence, Rhode Island, 1990.

32. Crank, J., The Mathematics of Diffusion, 414 pp., Clarendon Press, Oxford, 1975.

33. Cullinan, H. T. , Jr., Analysis of the flux equations of multicomponent diffusion, Ind. Engr. Chem. Fund., 4, 133-139, 1965.

3.1. Cygan, R. T., and Lasaga, A.
C., Self-diffusion of magnesium in garnet at $750-900^{\circ} \mathrm{C}, \mathrm{Am} . \mathrm{J}$. Sci., 285, 328-350, 1985.

35. Darken, L. S., Diffusion, mobility and their interrelation through free energy in binary metallic systems, Am. Inst. Min. Metal. Engrs. Trans., 175, 184-201, 1948.

36. de Groot, S. R., Mazur, P., Non-equilibrium Thermodynamics, 510 pp., North Holland, Amsterdam, 1962.

37. Dennis, P. F., Oxygen selfdiffusion in quartz under hydrothermal conditions, J. Geophys. Res., 89, 4047-4057, 1984.

38. Dodson, M. H., Closurc temperature in cooling geochronological and petrological problems, Contrib. Mineral. Petrol., 40, 259 274, 1973.

39. Draper, D. S., and Carroll, M. R., Diffusivity of Ar in haplobasaltic liquid at 10 to 30 kbar (abstract), AGU/ 1992 Fall Meeting, supplement to Eos Trans. $A G U, 73,642,1992$.

40. Dunn, T., Oxygen chemical diffusion in three basaltic liquids at elevated temperatures and pressures, Geochim. Cosmochim. Acta, 47, 1923-1930, 1983.

41. Elphick, S. C., Ganguly, J., and Loomis, T. P., Experimental determination of cation diffusivities in aluminosilicate garnets, I. Experimental methods and interdiffusion data, Contrib. Mineral. Petrol., 90, 36-44, 1985.

42. Elphick, S. C., Graham, C. M., and Dennis, P. F., An ion microprobe study of anhydrous oxygen diffusion in anorthite: a comparison with hydrothermal data and some geological implications, Contrib. Mineral. Petrol., 100, 490-495, 1988.

13. Farver, J. R., Oxygen self- diffusion in diopside with application to cooling rate determinations, Earth Planet. Sci. Lett., 92, 386-396, 1989.

44. Farver, J. R., and Giletti, B. J., Oxygen diffusion in amphiboles, Geochim. Cosmochim. Acta, 49, 1403-1411, 1985.

45. Farver, J. R., and Giletti, B. J., Oxygen and strontium diffusion kinetics in apatite and potential applications to thermal history determinations, Geochim. Cosmochim. Acta, 53, 1621-1631, 1989.

46. Farver, J. R., and Yund, R. A., The effect of hydrogen, oxygen, and water fugacity on oxygen diffusion in alkali feldspar, Geochim. Cosmochim. Acta, 54, 2953-2964, 1990.

47. Farver, J. R., and Yund, R. A., Measurement of oxygen grain boundary diffusion in natural, fine-grained quartz aggregates, Geochim. Cosmochim. Acta, 55, 1597-1607, 1991a.

48. Farver, J. R., and Yund, R. A., Oxygen diffusion in quartz: Dependence on temperature and water fugacity, Chem. Geol., 90, 55-70, 1991b.

49. Farver, J. R., and Yund, R. A., Oxygen diffusion in a finegrained quartz aggregate with wetted and nonwetted microstructures, J. Geophys. Res., 97, 14017-14029, 1992.

50. Fick, A., Über diffusion, Ann. Physik Chem., 94, 59, 1855.

51. Hisher, G. W., Nonequilibrium thermodynamics as a model for diffusion-controlled metamorphic processes, Am. J. Sci., 273, 897-924, 1973.

52. Foland, K. A., ${ }^{40} \mathrm{Ar}$ diffusion in homogencous orthoclase and an interpretation of Ar diffusion in K-feldspars, Geochim. Cosmochim. Acta, 38, $151-166$, $1974 a$. 
53. Foland, K. A., Alkali diffusion in orthoclase, in Geochemical Transport and Kinetics, edited by Hofmann, A. W., Giletti, B. J., Yoder, H. S., Jr., and Yund, R. A., pp. 77-98, Carnegie Institution of Washington, Washington, 1974b.

54. Fortier, S. M., and Giletti, B. J., Volume self-diffusion of oxygen in biotite, muscovite, and phlogopite micas, Geochim. Cosmochim. Acta, 55, 1319 1330, 1991.

55. Foster, C. T., A thermodynamic model of mineral segregations in the lower sillimanite zone near Rangeley, Maine, $A m$. Mineral., 66, 260-277, 1981.

56. Freer, R., Self-diffusion and impurity diffusion in oxides, $J$. Materials Sci., 15, 803-824, 1980.

57. Freer, R., Diffusion in silicate minerals and glasses: a data digest and guide to the literature, Contrib. Mineral. Petrol., 76, 440-454, 1981.

58. Freer, R., and Dennis, P. F., Oxygen diffusion studies. I. A preliminary ion microprobe investigation of oxygen diffusion in some rock-forming minerals, Mineral. Mag., 45, 179-192, 1982.

59. Freer, R., and Hauptman, Z., An experimental study of magnetite-titanomagnetite interdiffusion, Phys. Earth Planet. Inter., 16, 223-231, 1978.

60. Ganguly, J., and Tazzoli, V., $\mathrm{Fe}^{+2}-\mathrm{Mg}$ interdiffusion in orthopyroxene: constraints from cation ordering and structural data and implications for cooling rates of meteorites (abstract), in Lunar and Planet. Science $X X I V$, pp. 517-518, Lunar and Planet. Institute, Houston, 1992.

61. Gérard, O., and Jaoul, O.,
Oxygen diffusion in San Carlos olivine, J. Geophys. Res., 94, 41 19-4I28, 1989.

62. Giletti, B. J., Studies in diffusion I: argon in phlogopite mica, in Geochemical Transport and Kinetics, edited by Hofmann, A. W., Giletti, B. J., Yoder, H. S., Jr., and Yund, R. A., pp. 107-115, Carnegie Institution of Washington, Washington, 1974.

63. Giletti, B. J., $\mathrm{Rb}$ and $\mathrm{Sr}$ diffusion in alkali feldspars, with implications for cooling histories of rocks, Geochim. Cosmochim. Acta, 55, 1331 1343, 1991 .

64. Giletti, B. J., Diffusion kinetics of $\mathrm{Sr}$ in plagioclase (abstract), AGU 1992 Spring Meeting, supplement to Eos Trans. $A C U$, 73, 373, 1992.

65. Giletti, B. J., and Hess, K. C., Oxygen diffusion in magnetite, Earth Planet. Sci. Lett., 89, 115-122, 1988.

66. Giletti, B. J., Semet, M. P., and Yund, R. A., Studies in diffusion -- III. Oxygen in feldspars: an ion microprobe determination, Geochim. Cosmochim. Acta, 42, 45-57, 1978.

67. Giletti, B. J., and Tullis, J., Studies in diffusion, IV. Pressure dependence of $\mathrm{Ar}$ Diffusion in Phlogopite mica, Earth Planet. Sci. Lett., 35, 180-183, 1977.

68. Giletti, B. J., and Yund, R. A., Oxygen diffusion in quartz, $J$. Geophys. Res., 89, 4039-4046, 1984.

69. Giletti, B. J., Yund, R. A., and Semet, M., Silicon diffusion in quartz, Geol. Soc. Am. Abstr. Prog., 8, 883-884, 1976.

70. Graham, C. M., Experimental hydrogen isotope studies III: diffusion of hydrogen in hydrous minerals, and stable isotope exchange in metamorphic rocks, Contrib. Mineral. Petrol., 76, 216-228, 1981.

71. Graham, C. M., and Elphick, S. C., Some experimental constraints on the role of hydrogen in oxygen and hydrogen diffusion and Al-Si interdiffusion in silicates, in Diffusion, Atomic Ordering, and Mass Transport: Selected Problems in Geochemistry, edited by Ganguly, J., pp. 248-285, Springer-Verlag, New York, 1991.

72. Graham, C. M., Viglino, J. A., and Harmon, R. S., Experimental study of hydrogenisotope exchange between aluminous chlorite and water and of hydrogen diffusion in chlorite, Am. Mineral., 72, 566-579, 1987.

73. Griggs, D. T., Hydrolytic weakening of quartz and other silicates, Geophys. J., 14, 19 $31,1967$.

74. Grove, T. L., Baker, M. B., and Kinzler, R. J., Coupled CaAl$\mathrm{NaSi}$ diffusion in plagioclase feldspar: experiments and applications to cooling rate speedometry, Geochim. Cosmochim, Acta, 48, 2113-2121, 1984.

75. Gupta, P. K., and Cooper, A. R., Jr., The [D] matrix for multicomponent diffusion, Physica, 54, 39-59, 1971.

76. Harrison, T. M., Diffusion of ${ }^{40} \mathrm{Ar}$ in hornblende, Contrib. Mineral. Petrol., 78, 324-331, 1981.

77. Harrison, T. M., Duncan, I., and McDougall, I., Diffusion of ${ }^{40} \mathrm{Ar}$ in biotite: temperature, pressure and compositional effects, Geochim. Cosmochim. Acta, 49, 2461-2468, 1985.

78. Harrison, T. M., and Watson, 
E. B., Kinetics of zircon dissolution and zirconium diffusion in granitic melts of variable water content, Contrib. Mineral. Petrol., 84, 66-72, 1983.

79. Harrison, T. M., and Watson, E. B., The behavior of apatite during crustal anatexis: equilibrium and kinetic considerations, Geochim. Cosmochim. Acta, 48, 1467 1477, 1984.

80. Harrison, W. J., and Wood, B. J., An experimental investigation of the partitioning of REE between garnet and liquid with reference to the role of defect equilibria, Contrib. Mineral. Petrol., 72, 145-155, 1980.

81. Harrop, P. J., Self-diffusion in simple oxides (a bibliography), J. Materials Sci., 3, 206-222, 1968.

82. Hart, S. R., Diffusion compensation in natural silicates, Geochim. Cosmochim. Acta, 45, 279-291, 1981.

83. Hartley, G. S., and Crank, J., Some fundamental definitions and concepts in diffusion processes, Trans. Faraday Soc., 45, 801-818, 1949.

84. Hayashi, T., and Muehlenbachs, $K$., Rapid oxygen diffusion in melilite and its relevance to meteorites, Geochim. Cosmochim. Acta, 50, 585-591, 1986.

85. Hermeling, J., and Schmalzreid, $\mathrm{H}$., Tracerdiffusion of the $\mathrm{Fe}$ cations in Olivine $\left(\mathrm{Fe}_{\mathrm{x}} \mathrm{Mg}_{\mathrm{I}-\mathrm{x}}\right)_{2}$ $\mathrm{SiO}_{4}$ (III), Phys. Chem. Miner., 11, 161-166, 1984

86. Hofmann, A. W., Diffusion in natural silicate melts: a critical review, in Physics of Magmatic Processes, edited by Hargraves, R. B., pp. 385-417, Princeton Univ. Press, 1980.

87. Hofmann, A. W., and Giletti,
B. J., Diffusion of geochronologically important nuclides in minerals under hydrothermal conditions, Eclogae Geol. Helv., 63, 141-150., 1970.

88. Hofmann, A. W., Giletti, B. J., Hinthorne, J. R., Andersen, C. A., and Comatord, D., Ion microprobe analysis of a potassium self-diffusion experiment in biotite, Earth Planet. Sci. Lett., 24, 48-52, 1974.

89. Hofmann, A. W., and Magaritz, M., Diffusion of $\mathrm{Ca}, \mathrm{Sr}, \mathrm{Ba}$, and $\mathrm{Co}$ in a basalt melt, $J$. Geophys. Res., 82, 5432-5440, 1977.

90. Houlier, B., Cheraghmakani, M., and Jaoul, O., Silicon diffusion in San Carlos olivine, Phys. Earth Planet. Inter., 62, 329-340, 1990.

91. Jambon, A., Tracer diffusion in granitic melts, J. Geophys. Res., 87, 10797-10810, 1982.

92. Jambon, A., and Semet, M. P., Lithium diffusion in silicate glasses of albite, orthoclase, and obsidian composition: an ionmicroprobe determination, Earth Planet. Sci. Lett., 37, 445-450, 1978.

93. Jambon, A., Zhang, Y, and Stolper, E. M., Experimental dehydration of natural obsidian and estimation of $\mathrm{D}_{\mathrm{H} 2 \mathrm{O}}$ at low water contents, Geochim. Cosmochim. Acta, 56, 2931 2935, 1992.

94. Joesten, R., Evolution of mineral assemblage zoning in diffusion metasomatism, Geochim. Cosmochim. Acta, 41, 649-670, 1977.

95. Joesten, R., Grain-boundary diffusion kinetics in silicates and oxide minerals, in Diffusion, Atomic Ordering, and Mass Transport: Selected Problems in Geochemistry, edited by Ganguly, J., pp. 345-
395, Springer-Verlag, New York, 1991a.

96. Joesten, R., Local equilibrium in metasomatic processes revisited. Diffusion-controlled growth of chert nodule reaction rims in dolomite, $A m$. Mineral., 76, 743-755, $1991 \mathrm{~b}$.

97. Joesten, R., and Fisher, G., Kinetics of diffusion-controlled mineral growth in the Christmas Mountains (Texas) contact aureole, Geol. Soc. Am. Bull., 100, 714-732, 1988.

98. Johnson, C. D., and Carlson, W. D., The origin of olivineplagioclase coronas in metagabbros from the Adirondack Mountains, New York, $J$. Metamorph. Geol., 8, 697-717, 1990.

99. Jost, W., Diffusion in Solids, Liquids, and Gases, 558 pp., Academic Press, New York, 1960.

100. Jurewicz, A. J. G., and Watson, E. B., Cations in olivine, part 2: diffusion in olivine xenocrysts, with applications to petrology and mineral physics, Contrib. Mineral. Petrol., 99, 186-201, 1988.

101. Kasper, R. B., Cation and oxygen diffusion in albite, Ph.D. thesis, Brown University, Providence, Rhode Island, 1975.

102. Kingery, W. D., Plausible concepts necessary and sufficient for interpretation of grain boundary phenomena: I, grain boundary characteristics, structure, and electrostatic potential, $A m$. Ceram. Soc. J., 57, 1-8, 1974a.

103. Kingery, W. D., Plausible concepts necessary and sufficient for interpretation of grain boundary phenomena: II, solute segregation, grain boundary diffusion, and general discussion, Am. Ceram. Soc. J., 57, 74-83, 1974b. 
104. Kronenberg, A. K., Kirby, S. H., Aines, R. D., and Rossman, G. R., Solubility and diffusional uptake of hydrogen in quartz at high water pressures: implications for hydrolytic weakening, J. Geophys. Res., 91, 12723-12744, 1986.

105. Lasaga, A. C., Multicomponent exchange and diffusion in silicates, Geochim. Cosmochim. Acta, 43, 455-469, 1979.

106. Lasaga, A. C., The atomistic basis of diffusion: defects in minerals, in Kinetics of Geochemical Processes, edited by Lasaga, A. C., and Kirkpatrick, R. J., pp. 261-320, Mineralogical Society of America, Washington, 1981.

107. Lasaga, A. C., Geospeedometry: an extension of geothermometry, in Kinetics and Equilibrium in Mineral Reactions, edited by Saxena, S. K., pp. 81-114, SpringerVerlag, New York, 1983.

108. Lesher, C. E., and Walker, D., Thermal diffusion in petrology, in Diffusion, Atomic Ordering, and Mass Transport: Selected Problems in Geochemistry, edited by Ganguly, J., pp. 396451, Springer-Verlag, New York, 1991.

I(19. I in, T. H., and Yund, R. A., Potassium and sodium selfdiffusion in alkali feldspar, Contrib. Mineral. Petrol., 34, 177-184, 1972.

110. Lindner, R., Studies on solid state reactions with radiotracers, J. Chem. Phys., 23, 410-411, 1955.

111. Lindner, R., Silikatbildung durch Reaktion im festen Zustand, Z. Physik. Chem., 6, 129-142, 1956.

112. Lindström, R., Chemical diffusion in alkali halides, $J$. Phys. Chem. Solids, 30, 401-
405,1969

113. Lindström, R., Chemical interdiffusion in binary ionic solid solutions and metal alloys with changes in volume, $J$. Phys. $C$ : Solid State, 7, 3909-3929, 1974.

114. Liu, M., and Yund, R. A., $\mathrm{NaSi}-\mathrm{CaAl}$ interdiffusion in plagioclase, Am. Mineral., 77, 275-283, 1992.

115. Loomis, T. P., Ganguly, J., and Elphick, S. C., Experimental determination of cation diffusivities in aluminosilicate garnets II. Multicomponent simulation and tracer diffusion coefficients, Contrib. Mineral. Petrol., 90, 45-51, 1985.

116. Lowry, R. K., Henderson, P., and Nolan, J., Tracer diffusion of some alkali, alkaline-earth and transition element ions in a basaltic and an andesitic melt, and the implications concerning melt structurc, Contrib. Mineral. Petrol., 80, 254-261, 1982.

117. Lowry, R. K., Reed, S. J. B., Nolan, J., Henderson, P., and Long, J. V. P., Lithium tracerdiffusion in an alkali-basalt melt -- an ion-microprobe determination, Earth Planet. Sci. Lett., 53, 36-41, 1981.

118. Mackwell, S. I., and Kohlstedt, D. L., Diffusion of hydrogen in olivine: implications for water in the mantle, $J$. Geophys. Res., 95, 5079-5088, 1990.

119. Magaritz, M., and Hofmann, A. W., Diffusion of $\mathrm{Sr}, \mathrm{Ba}$, and $\mathrm{Na}$ in obsidian, Geochim. Cosmochim. Acta, 42, 595-605, 1978a.

120. Magaritz, M., and Hofmann, A. W., Diffusion of Eu and Gd in basalt and obsidian, Geochim. Cosmochim. Acta, 42, 847. 858,1978 b

121. Manning, J. R., Diffusion
Kinetics for Atoms in Crystals, 257 pp., Van Nostrand, Princeton, 1968.

122. Matano, C., On the relation between the diffusion coefficients and concentrations of solid metals, Japan J. Phys., 8, 109-113, 1933.

123. McDougall, I., Harrison, T. M., Geochronology and Thermochronology by the ${ }^{40} \mathrm{Ar} /{ }^{39} \mathrm{Ar}$ Method, 212 pp., Oxford Univ. Press, New York, 1988.

124. Meisne:, D. J., Cationic diffusion in olivine to $1400^{\circ} \mathrm{C}$ and 35 kbar, in Geochemical Transport and Kinetics, edited by Hofmann, A. W., Giletti, B. J., Yoder, H. S., Jr., and Yund, R. A., pp. 117-129, Carnegie Institution of Washington, Washington, 1974

125. Morioka, M., Cation diffusion in olivine -- I. Cobalt and magnesium, Geochim. Cosmochim. Acta, 44, 759-762, 1980.

126. Morioka, M., Cation diffusion in olivine -- II. Ni-Mg, $\mathrm{Mn}-\mathrm{Mg}$, $\mathrm{Mg}$ and $\mathrm{Ca}$, Geochim. Cosmochim. Acta, 45, 15731580, 1981.

127. Morioka, M., and Nagasawa, H., Diffusion in single crystals of melilite: II. Cations, Geochint. Cosmochim. Acta, 55, 751-759, 1991a

128. Morioka, M., and Nagasawa, $\mathrm{H}$., Ionic diffusion in olivine, in Diffusion, Atomic Ordering, and Mass Transport: Selected Problems in Geochemistry, edited by Ganguly, J., pp. 176197, Springer-Verlag, New York, $1991 \mathrm{~b}$.

129. Morishita, Y., Giletti, B. J., and Farver, J. R., Strontium and oxygen self-diffusion in titanite (abstract), Eos Trans. AGU, 7I, $652,1990$.

130. Muehlenbachs, K., and Connolly, C., Oxygen diffusion 
in leucite, in Stable Isotope Geochemistry: A Tribute to Samuel Epstein, edited by Taylor, H. P., Jr., O'Neil, J. R., and Kaplan, I. R., pp. $27-$ 34. The Geochemical Society, San Antonio, Texas, 1991.

131. Nye, J. F., Physical Properties of Crystals, 322 pp., Clarendon Press, Oxford, 1957.

132. Onsager, L., Reciprocal relations in irreversible processes, I, Physical Rev., 37, 405-426, 1931 a.

133. Onsager, L., Reciprocal relations in irreversible processes II, Physical Rev., 38, 2265 2279, 193 lb.

134. Onsager, L., Theories and problems of liquid diffusion, Ann. N.Y. Acad. Sci., 46, 241 265, 1945.

135. Philibert, Jean, Atom Movements: Diffusion and Mass Transport in Solids, 577 pp., Editions de Physique, Les Ulis, France, 1991.

136. Price, G. D., Diffusion in the titanomagnetite solid solution, Mineral. Mag., 44, 195-200, 1981.

137. Prigogine, I., Introduction to Thermodynamics of Irreversible Processes, 147 pp., Wiley Interscience, New York, 1967.

138. Rapp, R. A., and Watson, E. B., Monazite solubility an dissolution kinetics: implications for the thorium and light rare earth chemistry of felsic magmas, Contrib. Mineral. Petrol., 94, 304-316, 1986.

139. Reiss, Howard, Methods of Thermodynamics, 217 pp., Blaisdell Publishing Company, Waltham, Massachusetts, 1965.

140. Rovetta, M. R., Holloway, J. R., and Blacic, J. D., Solubility of hydroxl in natural quartz annealed in water at $900^{\circ} \mathrm{C}$ and $1.5 \mathrm{GPa}$, Geophys. Res. Lett.,
13, 145-148, 1986.

141. Ryerson, F. J., Diffusion measurements: experimental methods, Methods Exp. Phys., 24A, 89-130, 1987.

142. Ryerson, F. J., Durham, W. B., Cherniak, D. J., and Lanford, W. A., Oxygen diffusion in olivine: effect of oxygen fugacity and implications for creep, J. Geophys. Res., 94, 4105-4118, 1989.

143. Ryerson, F. J., and McKeegan, K. D., Determination of oxygen self-diffusion in åkermanite, anorthite, diopside, and spinel: Implications for oxygen isotopic anomalies and the thermal histories of $\mathrm{Ca}-\mathrm{Al}$-rich inclusions, Geochim. Cosmochim. Acta, 58(17), in press, 1994.

144. Sautter, V., Jaoul, O., and Abel, F., Aluminum diffusion in diopside using the ${ }^{27} \mathrm{Al}(\mathrm{p}, \gamma)^{28} \mathrm{Si}$ nuclear reaction: preliminary results, Earth Planet. Sci. Lett., 89, 109-114, 1988.

145. Shaffer, E. W., Shi-Lan Sang, J., Cooper, A. R., and Heuer, A. H., Diffusion of tritiated water in b-quartz, in Geochemical Transport and Kinetics, edited by Hofmann, A. W., Giletti, B. J., Yoder, H. S., Jr., and Yund, R. A., pp. 131-138, Carnegic Institution of Wash., Washington, 1974.

146. Sharp, Z. D., Giletti, B. J., and Yoder, H. S., Jr., Oxygen diffusion rates in quartz exchanged with $\mathrm{CO}_{2}$, Earth Planet. Sci. lett., I07, 339-348, 1991.

147. Shewmon, Paul G., Diffusion in Solids, 246 pp., Minerals, Metals \& Materials Society, Warrendale, PA, 1989

148. Smigelskas, A. D., and Kirkendall, E. O., Zinc diffusion in alpha brass, Am. Inst.
Min. Metal. Engrs. Trans., 171, 130-142, 1947.

149. Sneeringer, M., Hart, S. R., and Shimizu, N., Strontium and samarium diffusion in diopside, Geochim. Cosmochim. Acta, 48, 1589-1608, 1984.

150. Tiernan, R. J., Diffusion of thallium chloride into single crystals and bicrystals of potassium chloride, Ph.D. thesis, MIT, Cambridge, Massachusetts, 1969.

151. Trull, T. W., and Kurz, M. D., Experimental measurement of ${ }^{3} \mathrm{He}$ and ${ }^{4} \mathrm{He}$ mobility in olivine and clinopyroxene at magmatic temperatures, Geochim. Cosmochim. Acta, 57, 1313-1324, 1993.

152. Watson, E. B., Calcium diffusion in a simple silicate melt to 30 kbar, Geochim. Cosmochim. Acta, 43, 313322, 1979a.

153. Watson, E. B., Diffusion of cesium ions in $\mathrm{H}_{2} \mathrm{O}$-saturated granitic melt, Science, 205, 1259-1260, 1979 b.

154. Watson, E. B., Diffusion in fluid-bearing and slightly-melted rocks: experimental and numerical approaches illustrated by iron transport in dunite, Contrib. Mineral. Petrol., 107, 417-434, 1991a.

155. Watson, E. B., Diffusion of dissolved $\mathrm{CO}_{2}$ and $\mathrm{Cl}$ in hydrous silicic to intermediate magmas, Geochim. Cosmochim. Acta, 55, 1897-1902, 1991b.

156. Watson, E. B., and Baker, D. $R$., Chemical diffusion in magmas: an overview of experimental results and genchemical applications, in Physical Chemistry of Magmas, Advances in Physical Geochemistry, 9, edited by Perchuk, L., and Kushiro, I., pp. 120151, Springer-Verlag, New 
York, 1991.

157. Watson, E. B., Harrison, T. M., and Ryerson, F. J., Diffusion of $\mathrm{Sm}, \mathrm{Ar}$, and $\mathrm{Pb}$ in fluorapatite, Geochim. Cosmochim. Acta, 49, 1813-1823, 1985.

158. Watson, E. B., and Lupulescu, A., Aqueous fluid connectivity and chemical transport in clinopyroxene-rich rocks, Earth Planet. Sci. Lett., II7, 279 294, 1993.

159. Watson, E. B., Sneeringer, M. A., and Ross, A., Diffusion of dissolved carbonate in magmas: experimental results and applications, Earth Planet. Sci. Lett., 61, 346-358, 1982.

160. Wendlandt, R. F., Oxygen diffusion in basalt and andesite melts: experimental results and discussion of chemical versus tracer diffusion, contrib. Mineral. Petrol., 108, 463-471, 1991.

161. Wendt, R. P., The estimation of diffusion coefficients for ternary systems of strong and weak electrolytes, J. Phys. Chem., 69, 1227-1237, 1965.

162. Winchell, P., The compensation law for diffusion in silicates, High Temp. Sci., I, 200-215, 1969.

163. Yund, R. A., Diffusion in feldspars, in Feldspar Mineralogy, edited by Ribbe, P. H., pp. 203-222, Mineralogical Society of America, Washington, 1983.

164. Yund, R. A., Quigley, J., and Tullis, J., The effect of dislocations on bulk diffusion in feldspars during metamorphism, J. Metamorph. Geol., 7, 337 $341,1989$.

165. Yurimoto, H., Morioka, M., and Nagasawa, H., Diffusion in single crystals of melilite: I. oxygen, Geochim. Cosmochim. Acta, 53, 2387-2394, 1989.

166. Zhang, Y., Stolper, E. M., and Wasserburg, G. J., Diffusion in rhyolite glasses, Geochim. Cosmochim. Acta, 55, 441 456, 1991a.

167. Zhang, Y., Stolper, E. M., and Wasserburg, G. J., Diffusion of a multi-species component and its role in oxygen and water transport in silicates, Earth Planet. Sci. Lett., 103, 228 $240,1991 \mathrm{~b}$. 